\title{
Reduced Reach-Related Modulation of Motor Thalamus Neural Activity in a Rat Model of Parkinson's Disease
}

\author{
Clémentine Bosch-Bouju, ${ }^{1}$ Roseanna A. Smither, ${ }^{2}$ Brian I. Hyland, ${ }^{2}$ and Louise C. Parr-Brownlie ${ }^{1}$ \\ ${ }^{1}$ Department of Anatomy and ${ }^{2}$ Department of Physiology, Otago School of Medical Science, Brain Health Research Centre, University of Otago, Dunedin \\ 9054, New Zealand
}

\begin{abstract}
Motor thalamus (Mthal) is a key node in the corticobasal ganglia (BG) loop that controls complex, cognitive aspects of movement. In Parkinson's disease (PD), profound alterations in neuronal activity occur in BG nuclei and cortex. Because Mthal is located between these two structures, altered Mthal activity has been assumed to underlie the pathogenesis of PD motor deficits. However, to date, inconsistent changes in neuronal firing rate and pattern have been reported in parkinsonian animals. Moreover, although a distinct firing pattern of Mthal neurons, called low-threshold calcium spike bursts (LTS bursts), is observed in reduced preparations, it remains unknown whether they occur or what their role might be in behaving animals. We recorded Mthal spiking activity in control and unilateral 6-hydroxydopamine lesioned rats performing a skilled forelimb-reaching task. We show for the first time that Mthal firing rate in control rats is modulated in a temporally precise pattern during reach-to-grasp movements, with a peak at the time of the reach-end and troughs just before and after it. We identified LTS-like events on the basis of LTS burst characteristics. These were rare, but also modulated, decreasing in incidence just after reach-end. The inhibitory modulations in firing rate and LTS-like events were abolished in parkinsonian rats. These data confirm that nigrostriatal dopamine depletion is accompanied by profound and specific deficits in movement-related Mthal activity. These changes would severely impair Mthal contributions to motor program development in motor cortex and are likely to be an important factor underlying the movement deficits of PD.
\end{abstract}

Key words: electrophysiology; LTS bursts; motor thalamus; Parkinson's disease; reaching task

\section{Introduction}

Motor thalamus (Mthal) receives major input from basal ganglia (BG) and cerebellum (CB), as well as motor-related cortical areas, which are also its major target. Mthal is a key node in the corticobasal ganglia loop involved in the complex cognitive control of movement (Middleton and Strick, 2000, Bosch-Bouju et al., 2013). Consistent with this role, previous studies on Mthal activity during preparation and execution of normal movements found phasic increases in firing rate, as well as decreases and more complex modulations, time locked to various movement components (Strick, 1976; Schmied et al., 1979; Horne and Porter, 1980; MacPherson et al., 1980; Schlag-Rey and Schlag, 1984; Anderson and Turner, 1991, Nambu et al., 1991; Butler et al., 1992, 1996; Forlano et al., 1993; Vitek et al., 1994; Inase et al., 1996; Ivanusic et al., 2005; Kurata, 2005; Kunimatsu and Tanaka, 2010). In Parkinson's disease (PD), there are profound alterations in neuronal

\footnotetext{
Received March 5, 2014; revised 0ct. 1, 2014; accepted 0ct. 4, 2014.

Author contributions: B.I.H. and L.C.P.-B. designed research; C.B.-B. and R.A.S. performed research; C.B.-B., B.I.H., and L.C.P.-B. analyzed data; C.B.-B., B.I.H., and L.C.P.-B. wrote the paper.

This work was supported by grants from the Neurological Foundation of New Zealand (to L.C.P.-B.) and the Health Research Council of New Zealand (to B.I.H. and L.C.P.-B.).

The authors declare no competing financial interests.

Correspondence should be addressed to Dr. Louise Parr-Brownlie, Department of Anatomy, University of Otago, P0 Box 913, Dunedin 9054, New Zealand. E-mail: louise.parr-brownlie@otago.ac.nz.

C. Bosch-Bouju's present address: INRA, Nutrition and Integrative Neurobiology, UMR 1286, University of Bordeaux, 33076 Bordeaux, France.

DOI:10.1523/JNEUROSCI.0893-14.2014

Copyright $\odot 2014$ the authors $\quad 0270-6474 / 14 / 3415836-15 \$ 15.00 / 0$
}

activity in both BG nuclei and cortex (Obeso et al., 2000; Boraud et al., 2002; Goldberg et al., 2002; Parr-Brownlie and Hyland, 2005; Walters et al., 2007; Pasquereau and Turner, 2011) and, because Mthal is located between these structures, its activity is assumed to be altered and contribute to the pathogenesis of PD motor symptoms (Albin et al., 1989, Alexander and Crutcher, 1990a; DeLong, 1990; Galvan and Wichmann, 2008). However, to date, inconsistent changes in resting Mthal neuronal firing rate and pattern have been reported in parkinsonian animals (Voloshin et al., 1994; Schneider and Rothblat, 1996; Pessiglione et al., 2005). Further, although resting activity provides some indication of overall excitability in Mthal, the relationship of these changes to problems of movement generation in PD is not well understood.

To characterize Mthal movement-related activity and to determine the effects of that dopaminergic degeneration that occurs in PD, we recorded Mthal neuronal activity in control rats and rats with unilateral 6-hydroxydopamine (6-OHDA) lesions of the midbrain dopamine system. We show for the first time that specific components of complex reach-related modulations in Mthal activity are impaired by dopamine depletion.

\section{Materials and Methods}

Procedures. All procedures were approved by the University of Otago Committee on Ethics in Care and Use of Laboratory Animals and were in accordance with the National Institutes of Health's Principles of Laboratory Animal Care. Male Wistar rats $(180-250 \mathrm{~g}$ at the time of the first surgery) were housed in environmentally controlled conditions with a 
A
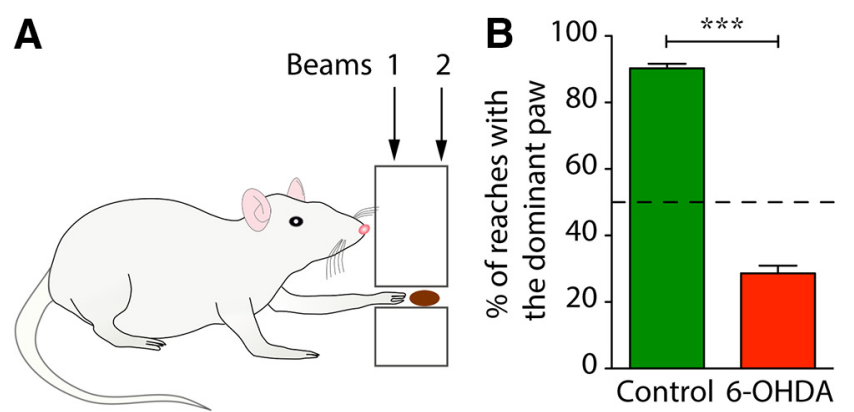

C

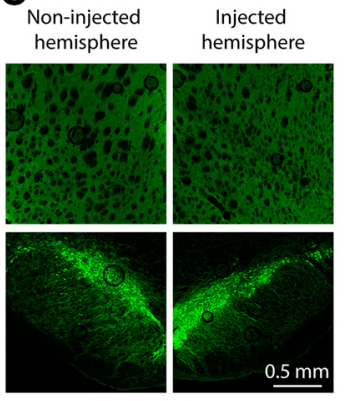

Control rats

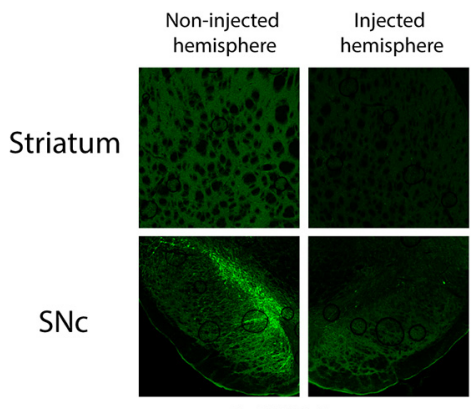

6-OHDA rats

Figure 1. The forelimb reaching task in control and 6-OHDA lesioned rats. A, Schematic of the forelimb reaching task. Infrared beams 1 and 2 spanned the reaching tube to allow detection of reaches and calculation of movement time. $\boldsymbol{B}$, Effect of unilateral 6-OHDA lesion on contralateral paw use in the reaching task. Rats were lesioned contralateral to the prelesion dominant paw ${ }^{* * *} p<0.0001$, Mann-Whitney test. C, Sections from striatum (top) and SNC (bottom) of example rats show immunohistochemical staining for TH (green) on the uninjected side of the brain (left) and from the side injected with vehicle (control rats) or 6-OHDA (6-OHDA rats).

reversed $12 \mathrm{~h}$ light/dark cycle and experiments were conducted during the animals' dark cycle. To ensure that rats were motivated to execute reaches with their forepaw, rats reached for palatable food and were limited to $15-18 \mathrm{~g}$ of standard rat chow every day in their home cage after the testing session. Rats were weighed weekly to ensure that their weight increased slowly or was maintained. Every effort was made to minimize the number of animals used.

Behavioral tasks. To assess reaching performance, rats were trained to reach into a 28 -mm-wide, 18 -mm-high rectangular opening positioned $55 \mathrm{~mm}$ above the floor of a Perspex box to retrieve chocolate-flavored breakfast cereal (Coco Pops; Kellogg's) placed on a tray attached to the exterior wall (Parr-Brownlie and Hyland, 2005; Fig. 1A). There was one training or testing session each day over which rats received $\sim 100$ pieces of cereal $(\sim 2.4 \mathrm{~g})$. Rats learned to reach for food with the paw after 2-3 sessions. The dominant paw was defined as the one used to execute reaches $>60 \%$ of the time (Fig. $1 B$ ). Movement time for the terminal part of each reach was derived from the time between interruption of two infrared light beams positioned $14 \mathrm{~mm}$ apart at $3 \mathrm{~mm}$ (beam 1) and 17 $\mathrm{mm}$ (beam 2) from the inner surface of the task box. Reaching in movement-related analyses was aligned to the time of interruption of beam 2 . The signals from each light beam interruption were converted to transistor-transistor logic (TTL) pulses and recorded with electrophysiological data using Spike2 (RRID: nlx_156886; Cambridge Electronic Design). Once the rats were trained, reaches made with the dominant paw were manually marked onto the data file using keystrokes. Annotating the use of the dominant paw allowed us to analyze spiking activity during reaches performed only with the dominant paw. For each experiment, 1 epoch (300 s) of reaching behavior was selected based on the presence of repeated TTL breaks and annotated notes indicating that the dominant paw was being used.

To better understand the physiology of Mthal in the control of movement, we also assessed open-field behaviors by placing rats into an openfield box for $30 \mathrm{~min}$. Periods of different behaviors in the open field were

noted by adding coded keystrokes on the neurophysiological recording files at the onset and offset of episodes of walking, rearing, or sniffing. For each experiment, 1 period (300 s) of electrophysiology data was selected based on offline video analyses.

Unilateral dopaminergic pathway lesions. Unilateral lesions of dopamine axons in the medial forebrain bundle were performed on rats weighing $180-250 \mathrm{~g}$ at the time of lesion surgery. Rats were fully anesthetized with a subcutaneous injection of ketamine ( $75 \mathrm{mg} / \mathrm{kg}$; PhoenixPharm Distributors), domitor (0.5 mg/kg; Pfizer), and atropine (0.06 $\mathrm{mg} / \mathrm{kg}$; PhoenixPharm Distributors). Body temperature was maintained at $36-38^{\circ} \mathrm{C}$ throughout the procedure by a heating pad. The incision area was shaved and a long-acting local anesthetic (Lignocaine 2\% gel; Orion Laboratories) was injected along the intended incision line. Rats were injected with desmethylimipramine ( $15 \mathrm{mg} / \mathrm{kg}$, i.p.; Sigma-Aldrich) 30 min before the neurotoxic lesion to prevent degeneration of noradrenergic neurons. Rats were mounted in a stereotaxic frame (Stoelting) and ophthalmic ointment (eyelube Tricin; Jurox) was applied to the eyes to prevent corneal dehydration. The skull was exposed by a midline sagittal incision and subcutaneous connective tissue was removed. A hole was drilled over the chosen stereotaxic coordinates above the medial forebrain bundle and the dura was reflected. For each rat, the hemisphere controlling their dominant paw (the contralateral one) was chosen for the lesion. Target coordinates for the cannula were centered for the medial forebrain bundle at anteroposterior (AP) $+4.4 \mathrm{~mm}$ from the lambdoid suture and lateral $-1.2 \mathrm{~mm}$ from lambda in the flat skull position and the cannula was lowered $8.3 \mathrm{~mm}$ from the skull surface. In lesioned rats, a solution of $6 \mu \mathrm{g}$ of 6 -OHDA in $3 \mu \mathrm{l}$ of $0.9 \%$ saline solution containing $0.008 \%$ ascorbic acid (Sigma-Aldrich) was infused at $1 \mu \mathrm{l} /$ min via a 26 gauge cannula. Control rats were sham lesioned with the same volume of vehicle. After the infusion, the cannula remained at the target site for an additional 5 min to prevent diffusion of the neurotoxin along the injection track. The incision was sutured, $5 \mathrm{mg} / \mathrm{kg}$ of the longacting analgesic carprofen (Carprieve; Norbrook), $5 \mathrm{ml}$ of $0.9 \%$ saline, and $2.5 \mathrm{mg} / \mathrm{kg}$ of the anesthesia reversal agent antisedan (Pfizer) were injected subcutaneously. Animals were checked postoperatively twice a day for 1 week after the surgery.

The extent of the dopaminergic denervation was tested behaviorally 3-4 d after the lesion using the step test (Olsson et al., 1995). Only rats that demonstrated a strong lesion effect (number of steps by contralateral limb/number of steps by ipsilateral limb $<0.05$ ) were used for electrophysiological recordings. At the end of the experiment, the extent of the lesion was further verified histologically (Fig. $1 C$, see Histology section).

Electrode implantation. Two weeks after the 6-OHDA or sham lesion, a bundle of 8 extracellular recording electrodes was implanted into the Mthal contralateral to the dominant paw of each rat. Electrodes were constructed from 0.0007 inch nichrome wire covered in formvar (A-M Systems). One end of each wire was stripped of the formvar covering and soldered into a gold amphenol pin (Connector Systems), which was inserted into a 9-way mini-connector (Science Technology Centre, Carleton University, Canada). The 8 wires were gathered into a bundle and the bundle was inserted into a 30 gauge stainless steel cannula so that the tips protruded $\sim 0.5 \mathrm{~mm}$ from the cannula. Each wire was trimmed with a fresh scalpel blade and the bundle was glued in place with cyanoacrylate (Supaglue; Selleys Chemical). Electrodes were gold plated for $3 \mathrm{~s}$ with a negative current of $10 \mu \mathrm{A}$ in a gold solution (5355; SIFCO Selective Plating) containing multiwalled carbon nanotubes ( $<8 \mathrm{~nm}$ diameter; Cheap Tubes; Ferguson et al., 2009). Final electrode impedances were between 0.1 and $0.9 \mathrm{M} \Omega$. The electrodes were attached to a microdrive that allowed the electrodes to be advanced through a vertical dorsoventral track in the Mthal in steps of $\sim 35 \mu \mathrm{m}$. A tinned copper earth wire (0.152 mm; RS Components) was soldered into a gold amphenol pin at one end, which was inserted into the mini-connector, and the other end was soldered to a stainless steel screw (Lomatt) that served as the earth screw. Electrode implantation was performed under full anesthesia following the same general procedures as 6-OHDA and sham lesions. Five stainless steel screws were implanted into the skull and a hole was drilled in the skull at the chosen stereotaxic coordinates above the Mthal (bregma AP $-2.0 \mathrm{~mm}$, lateral $1.6 \mathrm{~mm}$ ). The electrode assembly was lowered $-5 \mathrm{~mm}$ ventral to the surface of the brain. The earth screw was 
implanted on the skull above the contralateral CB. The mini-connector, the Microdrive, and a protective box with holes in the top to permit access to the microdrive for advancing the electrodes were fixed on the rat's skull with dental acrylic (Vertex-Dental; AA Zeist).

Single-unit recordings. All recordings were conducted in freely moving rats starting 7-10 d after the second surgery. During recording sessions, action potentials were amplified $(2000 \times)$ and band-pass filtered (154$9000 \mathrm{~Hz}$ ) using a CyberAmp signal conditioner (Molecular Devices). For each recording, activity on the earth wire served as the reference signal for all other wires. Channels with distinguishable single-cell activity were recorded using a Power1401 A-D converter and Spike2 (version 7) data acquisition and analysis software (Cambridge Electronic Design) at 40 $\mathrm{kHz}$. An amplitude threshold was set using the software to exclude most background noise and only spikes exceeding this were digitized, time stamped, and stored to disk. Extracellular action potentials from different single neurons were discriminated from each other and from noise offline, initially using the waveform shape template-matching algorithm in the Spike2 software and refined if necessary by subsequent cluster analysis on waveform principal components. Discrimination of spikes from noise was confirmed by the presence of an empty first bin in interspike interval (ISI) histograms calculated for each template (bin width, 1 $\mathrm{ms}$; time base, $1 \mathrm{~s}$ ). For each discriminated neuron, the waveform peakto-peak amplitude and duration were measured from the average calculated from all spikes recorded over the $300 \mathrm{~s}$ period of time analyzed for reaching or open-field periods.

Data analysis. We performed two types of analyses to characterize Mthal neural activity. First, we analyzed mean firing rate and measures of burst firing averaged over extended time periods during which the rats were either engaged in open-field behaviors (including walking, rearing, and sniffing) or performing repetitive reaching movements to retrieve and eat food rewards. Second, we analyzed specific modulations associated with each reach performed during the reaching task.

For overall measures of neuronal activity, 1 epoch (300 s) of recording that was free of artifact was used from each recorded neuron during open-field or reaching periods. Data are presented as means \pm SEM. Mean firing rate was determined as the total number of recorded spikes divided by the total time and expressed as spikes/second. We characterized cells as having bursty or nonbursty firing patterns using the discharge density histogram method as described previously (Kaneoke and Vitek, 1996; Parr-Brownlie et al., 2007). This analysis uses a statistical approach to define periods within a spike train that have a significantly higher firing rate than the overall mean (Kaneoke and Vitek, 1996). A burstiness index of 0.5 was used, which meant that the bin width of the density discharge histogram was set at twice the mean ISI for each recording. A spike train was classified as bursty if it met the following conservative criteria: there were at least 3 spikes in the bursts, the distribution of its discharge density histogram was significantly different from a Poisson distribution of the discharge density histogram $\left(\chi^{2}\right.$ test set at a significance level of 0.01 ), the histogram was positively skewed, the firing rate was $>1.0 \mathrm{~Hz}$ to accurately assess the distribution, and the number of bursts per 1000 spikes was $>5$ to have a minimum number of bursts across neurons that varied in firing rate (Parr-Brownlie et al., 2007, 2009; Walters et al., 2007).

Further, we searched for events that matched the characteristics of low threshold calcium spike (LTS) bursts in the spike trains. LTS bursts are a feature of thalamic relay neurons that have very specific underlying electrophysiological mechanisms requiring de-inactivation of T-type calcium channels by sustained hyperpolarization of the membrane potential and trigger very high intraburst spiking frequencies (Llinás and Jahnsen, 1982; Jahnsen and Llinás, 1984; Steriade et al., 1991). These mechanisms lead to specific temporal dynamics of LTS bursts compared with non-LTS bursts. It is impossible to identify LTS bursts definitively in extracellular recordings in freely moving animals because membrane potential and ion channel activation cannot be measured directly. Instead, criteria are set for variables that are thought to reflect the underlying physiological properties to exclude burst events that are unlikely to be LTS bursts. The remaining events, which meet the criteria, are referred to here as LTS-like events. After recent published studies that reported properties of putative LTS bursts (Lu et al., 1992; Parr-Brownlie et al.,
2009; Nakamura et al., 2014), we classified a burst as a LTS-like event if the first ISI in the event was $\leq 5 \mathrm{~ms}$, subsequent ISIs were $\leq 10 \mathrm{~ms}$ (i.e., minimum intraburst instantaneous frequency $=100 \mathrm{~Hz}$ ), and the event was preceded with a silent period of at least $100 \mathrm{~ms}$ (Lu et al., 1992; Lacey et al., 2007; Parr-Brownlie et al., 2009; Nakamura et al., 2014). The second criterion serves as a proxy for the hyperpolarization required to de-inactivate the T-type calcium channels and is likely to be conservative. We included doublets ( 2 spikes with ISI $\leq 5 \mathrm{~ms}$ ) within the category of LTS-like events.

For analyses of reach-related modulations, we recorded sufficient data to obtain at least 50 individual reaches with the dominant paw, usually achieved within a $300-600$ s period of reaching behavior. To detect and quantify reach-related modulations in firing rate and LTS-like events, we constructed separate peri-event time histograms (PETH, bin width 10 ms; Spike2) for spikes and for LTS-like events. The PETHs were aligned to the time that the dominant paw broke the infrared beam 2, marking the end of the extension phase of the reach and beginning of food grasping (Hyland and Jordan, 1997; Fig. 1A). PETH bin counts were converted to instantaneous frequency to normalize for different trial numbers. Because we annotated the recordings online with keystrokes to indicate those reaches performed with the prelesion dominant paw, we were able to separately analyze these reaches; that is, the paw controlled by the lesioned hemisphere in 6-OHDA rats.

For each PETH, the baseline was defined as the average spikes/second or LTS bursts/second during the period -0.6 to $-0.4 \mathrm{~s}$ before the light beam crossing. Bin values in PETH were standardized by converting to $z$-scores, where for each bin $i, \mathrm{Z}_{i}=\left(F_{\mathrm{i}}-F_{\mathrm{B}}\right) / \mathrm{SD}_{\mathrm{B}}$ where $F$ is the instantaneous frequency and $B$ is the baseline mean. Examination of the firing rate during reaching in control rats indicated that there were 5 common time epochs relative to reach-end during which many Mthal neurons showed similar firing rate modulations: in chronological order, we defined an initial peak epoch (P1) from -2.95 to $-0.60 \mathrm{~s}$, an initial trough (T1) from -0.19 to $-0.07 \mathrm{~s}$, a second peak (P2), from -0.04 to $0.02 \mathrm{~s}$, a second trough (T2) from 0.06 to $0.16 \mathrm{~s}$, and a third trough (T3) from 0.60 to $2.95 \mathrm{~s}$. The net area under the normalized PETH curves for firing rate and LTS-like event occurrence during these 5 defined epochs was calculated to quantify reach-related excitations and inhibitions in control and 6-OHDA lesioned rats. For this, we computed the net area between the curve and the zero $z$-score line for each epoch and each neuron, adding both positive and negative values, using Prism 5.0 software (RRID: nlx_156835; GraphPad). Results represent the average for each period of all neurons from control and 6-OHDA rats. Color plots of PETHs were generated with Igor Pro 6.32A (RRID: nlx_156887; Wavemetrics).

Statistical analyses. Statistical analyses were performed using GraphPad Prism 5.0 software. Multiple comparisons were made with one- or twofactor parametric or nonparametric ANOVA, as appropriate, with post hoc Bonferroni's or Dunnett's tests. Single comparisons were made with Student's $t$, Mann-Whitney, or Wilcoxon signed-rank tests and proportions were compared using Fisher exact tests or $\chi^{2}$ tests when $>2$ groups.

Histology. After completing recordings, rats were anesthetized with pentobarbital (100 mg/kg, i.p.) and recording sites marked by passing a positive current of $20 \mu \mathrm{A}$ for $30 \mathrm{~s}$ through 1 or 2 of the microwires that recorded the largest number of neurons. Rats were perfused intracardially with $400 \mathrm{ml}$ of $10 \%$ sucrose solution in phosphate buffer (PBsucrose) followed by $400 \mathrm{ml}$ of $4 \%$ paraformaldehyde in PBS. To maximize fixation, brains were extracted from the skull 3-4 h after the perfusion, postfixed in paraformaldehyde solution overnight, and then immersed in $30 \%$ sucrose in $\mathrm{PB}(0.1 \mathrm{M}, \mathrm{pH} 7.4)$ for at least $48 \mathrm{~h}$. Coronal sections of $40 \mu \mathrm{m}$ were collected with a microtome (Leica) and stored at $-20^{\circ} \mathrm{C}$. Three sequential slices in the region of the recording electrodes were stained; one slice was processed for neutral red, vesicular glutamate transporter 2 (VGluT2), or glutamate decarboxylase (67 kDa isoform, GAD67) stains. Sections used for neutral red staining were mounted on glass slides, incubated for $18 \mathrm{~min}$ in neutral red solution (FD Technologies), dehydrated, and coverslipped with DPX mountant (VWR International).

Sections through the midbrain were stained for tyrosine hydroxylase (TH) to visualize the effect of 6-OHDA lesion. Sections stained for VGluT2, GAD67, or TH were washed 3 times in $0.1 \mathrm{~m}$ PBS with the $\mathrm{pH}$ 




Figure 2. Location of recording electrode tracks in the Mthal. $\boldsymbol{A}$, Dots on atlas sections at different anteroposterior levels of Mthal (Paxinos and Watson, 2007) show the position of terminal electrolytic lesions made at the end of recording tracks from control (green) and 6-OHDA lesioned animals (red). Vertical lines show the track and horizontal lines show the calculated point from which recordings began or began to be included in the analysis if the first recordings were made dorsal to the Mthal. Shading indicates approximate positions of CB-receiving (VL, gray) and BG-receiving (VA, green) territories of Mthal, determined by GAD67 $(\boldsymbol{B})$ and VGluT2 staining (data not shown). $\boldsymbol{B}$, Representative histological sections at approximate levels shown in $\boldsymbol{A}$ stained for GAD67 (green). BG territory has dense immunohistochemical staining for GAD67 compared with CB territory. CB- and BG-receiving territories are delineated with white dashed lines. White arrows indicate the end of recording tracks.

adjusted to 7.4 and then 2 times in $0.2 \%$ Triton-PBS solution. Sections were incubated for $1 \mathrm{~h}$ in a solution containing $10 \%$ normal goat serum (Hercus Taieri Research Unit) and $0.2 \%$ Triton-PBS and then incubated with the primary antibody overnight at $4^{\circ} \mathrm{C}$. Sections were rinsed 3 times in $0.2 \%$ Triton-PBS solution, then incubated with the secondary antibody for $4 \mathrm{~h}$ at room temperature. Slices were mounted on slides with ProGold anti-Fade mountant with DAPI (Invitrogen) and visualized under a fluorescence microscope (Figs. 1D, 2B).

Primary antibodies used were as follows: rabbit polyclonal anti-TH antibody, 1:300 dilution (Millipore catalog \#AB152, RRID: AB_390204); rabbit polyclonal anti-VGluT2, 1:2500 dilution (Synaptic Systems, cata$\log$ \#135 403, RRID: AB_2254574); mouse monoclonal anti-GAD67, 1:1000 dilution (Millipore, catalog \#MAB5406; RRID: AB_2278725). Secondary antibodies were: goat anti-rabbit DyLight 488, 1:1000 dilution (Vector Laboratories, catalog \#DI-2488, RRID: AB_2307439) and goat antimouse Alexa Fluor 488, 1:1500 dilution (Invitrogen, catalog \#A11029, RRID: AB_138404).

\section{Results}

\section{Reaching performance in 6-OHDA lesioned rats}

We selected 6-OHDA lesioned rats $(n=4)$ that had a marked lesion-induced deficit in the use of their previously preferred paw, as confirmed by the step test (see Materials and Methods). These selected rats had an average step ratio (steps with previously dominant paw divided by steps with previously nondominant paw) of $0.02 \pm 0.01$. In contrast, control (sham-lesioned) rats had an average ratio of $0.81 \pm 0.17$ ( $n=6$ rats). Immunohistochemical staining for $\mathrm{TH}$ showed almost complete loss of dopamine neurons in the SNc and their terminals in the striatum in 6-OHDA lesioned rats (Fig. 1C).

Rats began experiments after both the lesion and electrode implantation surgical procedures, which corresponded to 3-4 and 1-2 weeks after these respective surgeries. Successful comple- tion of the reach-to-grasp movement requires precise orchestration of proximal and distal muscle activities (Hyland and Jordan, 1997). In particular, at reach-end, there is coordinated arrest of the forelimb extension, an "arpeggio" complex (forelimb pronation and digit extension) that positions the paw over the food, grasping movements of the digits, and then paw withdrawal (Whishaw et al., 1994). During the first week of experiments after surgery, lesioned rats increasingly used their nondominant paw to reach for the food. To motivate rats to keep using their prelesion dominant paw, we delivered the food pellet reward only when they performed at least one reach within a sequence with that paw. Before the lesion, rats on average executed $317 \pm 31$ reaches with either paw in $5 \mathrm{~min}$, whereas after the lesion, it fell to $182 \pm 8$ reaches $(n=4$ rats, $p=0.011$, unpaired $t$ test). In addition, over the $2-5$ month postsurgery experimental period, 6-OHDA rats performed an average of $28.6 \pm 2.3 \%$ of reaches with their prelesion dominant paw, whereas control rats used their dominant paw for $90.3 \pm 1.4 \%$ of reaches $(n=36$ and 73 experiments, respectively, $p<0.0001$; Fig. $1 B$ ). Interestingly, although rats executed fewer reaches with their dominant (affected) paw, a one-factor ANOVA indicated that the duration of the terminal part of the reach, measured as the time between the interruption of two infrared beams (Fig. 1A), did not differ between control and 6-OHDA rats for the dominant paw (23.2 \pm 0.7 and $22.2 \pm 1.2 \mathrm{~ms}$, respectively, $p=0.4942$, one-factor ANOVA). This lack of bradykinesia but clear akinesia in the unilateral 6-OHDA rat model of $\mathrm{PD}$ is consistent with findings by Whishaw et al. (1994) and suggests that 6-OHDA rats can behaviorally partially compensate for their lesion. These results may also reveal paradoxical kinesia, which is seen in PD patients (Wilson, 1925; Denny-Brown, 1968; Glickstein and Stein, 1991). In addition, for the nondominant paw, 6-OHDA lesioned rats had significantly shorter reaching times than control rats $(20.3 \pm 1.3 \mathrm{~ms}, n=36$ experiments vs $25.7 \pm 1.0 \mathrm{~ms}, n=53$ experiments, respectively, $p=0.0028$, one-factor ANOVA), perhaps because of their reliance on this paw both for executing reaches and for general movements, with higher level of use producing a practice effect. Therefore, consistent with a previous study (Whishaw et al., 1994), 6-OHDA lesioned rats were able to execute reaches with their impaired dominant paw, but did so less frequently, which is similar to the paucity of movement (akinesia) seen in human PD.

\section{Spiking activity in the Mthal during open field and reaching} We recorded neural activity from 6 control (123 neurons) and 4 6-OHDA lesioned rats (65 neurons). Only recordings confirmed to be in the Mthal were included in the study (Fig. 2A). We used a combination of GAD67 (Fig. 2B) and VGluT2 (data not shown) immunohistochemical staining to determine whether recorded neurons were located in ventroanterior (VA) (high level of GAD67 staining, low level of VGluT2 staining) or ventrolateral (VL) Mthal (lower level of GAD67 staining, higher level of 
VGluT2 staining; Kuramoto et al., 2011; Nakamura et al., 2014). This chemical segregation is explained by the high density of GABAergic inputs from the BG in the VA territory compared with the high density of glutamatergic inputs from the $\mathrm{CB}$ (and a low density of GABAergic inputs from the globus pallidus internus) in the VL territory. Therefore, the terms BG and $\mathrm{CB}$ territories of the Mthal were chosen to describe the areas recorded in the Mthal for the rest of the study. Although some of the more dorsal recording sites corresponded to the CB territory (VL), all tracks ended in and included recordings from the ventral part of the Mthal, so the majority $(143 / 188,76 \%)$ of recorded neurons were located in the BG territory (VA) of the Mthal.

To investigate the effect of 6-OHDA lesion on Mthal activity during different behavioral tasks, neuronal activity was recorded while rats moved about in an open field and during the performance of a specific and highly skilled reaching task that has functional correlates to reaching in humans (Whishaw et al., 1992). Figure $3 A$ shows example recordings obtained during open-field behavior, along with average waveforms of the recorded neurons. The extracellular action potentials recorded from control and 6-OHDA rats were not significantly different in peakto-peak duration (respectively, $0.65 \pm$ $0.02 \mathrm{~ms}, n=43$, vs $0.59 \pm 0.03 \mathrm{~ms}, n=24$, $p=0.1165$, unpaired $t$ test) or peak-topeak amplitude (respectively, $95 \pm 2 \mu \mathrm{V}$ vs $100 \pm 5 \mu \mathrm{V}, p=0.7191)$.

\section{Firing rate}

The analysis of overall neuronal firing rate during open-field behavior or while performing repeated reaching movements is shown in Figure 3B. Mthal neurons from control rats during open-field behavior and reaching displayed spiking frequencies from 0.22 to 9.43 spikes/s, with an average rate of $2.84 \pm 0.16$ spikes/s $(n=$ 123). The effect of 6-OHDA lesion on mean firing rate during open-field and reaching experiments is also shown in Figure $3 B$. A two-factor ANOVA with behavior (open-field, reaching) and group (control, 6-OHDA lesion) as factors revealed significant main effects of group $\left(F_{(1,183)}=\right.$ 11.97, $p=0.0007)$ and behavior $\left(F_{(1,183)}=10.98, p=0.0011\right)$, with no group $\times$ behavior interaction. Therefore, overall firing rate during reaching $(3.63 \pm 0.19$ spikes/s, $n=121)$ was higher than during open-field behavior $(2.34 \pm 0.29$ spikes $/ \mathrm{s}, n=66)$. This could simply reflect the presence of reach-related activity in these spike trains, which is addressed in the next section. More importantly, firing rates of Mthal neurons in 6-OHDA rats (3.88 \pm 0.37 spikes/s, $n=64)$ were significantly higher than in control rats $(2.82 \pm 0.15$ spikes/s, $n=123)$. Therefore, these data
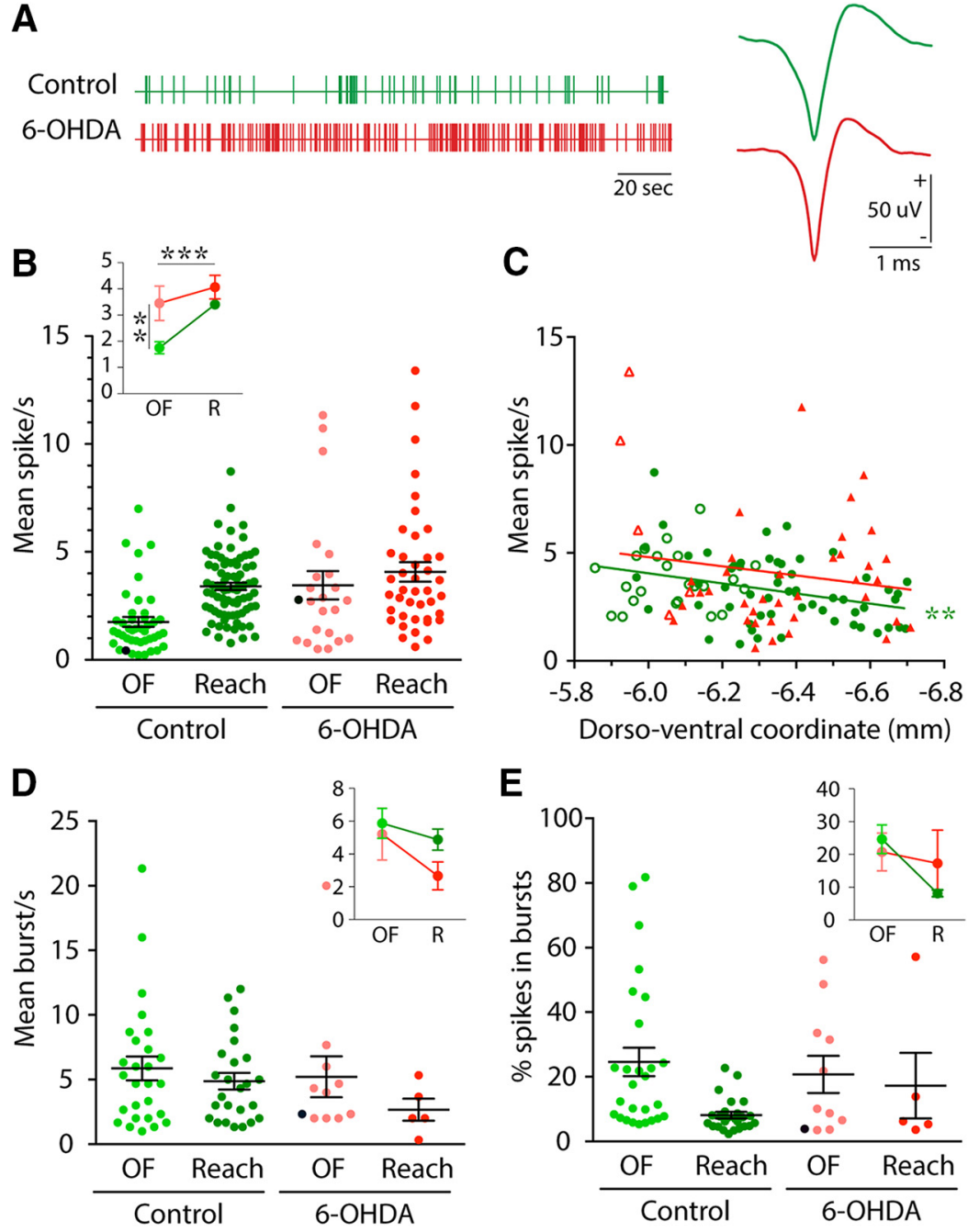

Figure 3. Effect of 6-OHDA lesion on neural activity in different behavioral tasks. $\boldsymbol{A}$, Vertical lines show single action potentials in example spike trains from neurons recorded in control (green) and 6-OHDA (red) rats during $200 \mathrm{~s}$ of open-field behavior. Representative action potentials are shown (right) for control (green) and 6-0HDA (red) rats. $\boldsymbol{B}$, Dots on the scatter plot show mean firing rate for each neuron recorded in control (green) and 6-OHDA (red) rats during open field (OF, light colors) and reaching (Reach, dark colors). Black lines represent grand mean \pm SEM over all neurons. Black dots show cells illustrated in $\boldsymbol{A}$. Interaction plot (inset) shows grand mean data comparing control and 6-OHDA lesioned groups during OF and reaching (R) behavioral tasks (same color code as scatter plot), ${ }^{* * *} p<0.001{ }^{* *} p<0.01$, for main effects of behavior (horizontal statistics) and group (vertica statistics), respectively. C, Scattergram of the dorsoventral location of recorded neurons plotted against their average firing rate during reaching. Data for control and 6-OHDA rats are represented in green and red, respectively. Filled symbols represent neurons recorded in BG territory and open symbols represent neurons recorded in CB territory of Mthal. Linear regressions are represented

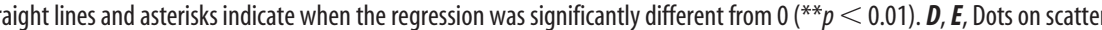
plots show mean burst occurrence $(\boldsymbol{D})$ and percentage of all spikes in bursts $(\boldsymbol{E})$ for each neuron recorded in control (green) and 6-OHDA (red) rats during OF (light colors) and Reach (dark colors) tasks. Black lines represent grand mean \pm SEM over all neurons. Black dots show cells illustrated in $\boldsymbol{A}$. Interaction plots (inset) show grand mean data comparing control and 6-OHDA lesioned groups, during $0 \mathrm{~F}$ and reaching (R) behaviors (same color code as scatter plot).

indicate that dopamine depletion consistently increased the mean firing rate of Mthal neurons whether animals were engaged in general behavior in an open field or performing a highly discrete skilled forelimb movement.

To investigate whether there were any differences in overall firing rate among neurons that may reflect different inputs of more dorsal CB-receiving and ventral BG-receiving territories, we performed a linear regression analysis of firing rate against dorsoventral location for data obtained during reaching. Allocation of individual neurons to one or other territory suggested that neurons in the BG territory (Fig. $3 C$, filled symbols) occurred at all depths, whereas neurons in the $\mathrm{CB}$ territory (Fig. $3 C$, open 
symbols) were found only in the dorsal half of electrode tracks. However, for quantitative analysis, we chose the conservative approach of using dorsoventral location, rather than the assigned thalamic nucleus, because of the difficulty to define the precise nuclear boundaries postmortem. For control rats (Fig. 3C, green symbols), there was a significant relationship between their dorsoventral location in Mthal and firing rate during reaching, with neurons situated more ventrally in the BG territory tending to have a lower rate $\left(r^{2}=0.12, p=0.0018\right)$. Conversely, for 6 -OHDA rats (red symbols), there was no significant relationship between firing rate and recording location $\left(r^{2}=0.03, p=0.32\right)$ even though recording sites were similar to control rats. This lack of relationship in 6-OHDA rats may indicate loss of specificity of responses in the Mthal during execution of the reaching task, as explored in the section Modulation of Mthal activity during reaching below.

\section{Bursting activity}

In addition to mean firing rate, firing pattern is functionally important, particularly clustering of spikes into groups with short ISIs known as "bursts." We characterized bursts in two ways; for one analysis, we identified all clusters of spikes in which the ISI stood out statistically from the background level using the Kaneoke and Vitek (1996) algorithm (see Materials and Methods). We refer to these as "general bursts." In another analysis, we identified all clusters of spikes that met specific criteria widely used in previous extracellular recording studies for identifying putative LTS bursts (Lu et al., 1992; Lacey et al., 2007; ParrBrownlie et al., 2009; Nakamura et al., 2014).

\section{General bursting}

To determine whether the burst firing pattern varied with behavioral state and if it was affected by 6-OHDA lesion, we performed separate two-way ANOVAs with factors of group (control, lesioned) and behavior (open-field, reaching) on two measures, the burst rate (Fig. 3D) and the percentage of all spikes in bursts (Fig. $3 E$ ). In a separate analysis, we examined the total number of cells that exhibited bursting. With the number of cells available, no significant difference was detected between reaching and open field or between control and lesioned values for either burst rate (Fig. 3D) or percentage spikes in bursts (Fig. 3E). However, despite this, fewer neurons were classified as bursty during reaching than in open field in control (respectively, $31.65 \%$ of neurons vs $62.79 \%, p=0.0011$, Fisher's exact tests) and 6-OHDA lesioned rats (respectively, $11.9 \%$ vs $47.83 \%, p=0.0008$ ), which is in contrast to the changes in firing rate. Importantly, the effect of 6-OHDA lesion was also in the opposite direction to that for firing rate, with fewer neurons classified as bursty during reaching in lesioned rats than in controls $(p=0.0258$, Fisher's exact test). Therefore, in Mthal, chronic dopamine depletion tends to be associated with an increase in mean firing rate, but a decrease in the proportion of bursty neurons in freely moving rats $(24.6 \%$ vs $42.6 \%$ in control rats, $p=0.0148$, Fisher's exact test).

\section{LTS-like events}

It has been hypothesized previously that dopamine depletion would result in more LTS bursts in the Mthal (Paré et al., 1990; Jeanmonod et al., 1996; Magnin et al., 2000; Guo et al., 2008). This hypothesis arises because the synchronized GABAergic activity in BG output nuclei in animal models of PD (Boraud et al., 2002; Walters et al., 2007) may hyperpolarize Mthal neurons (Person and Perkel, 2005, 2007; Kojima and Doupe, 2009; Leblois et al., 2009) and thus de-inactivate T-type calcium channels, which is required for LTS burst generation (Llinás and Jahnsen,
1982; Jahnsen and Llinás, 1984). However, data are not completely in support of this hypothesis. Of two studies in PD patients, one reported that many thalamic neurons exhibit putative LTS bursts (Magnin et al., 2000), whereas the other found that very few neurons had such activity (Zirh et al., 1998). These studies in human PD were of course not able to be compared with healthy controls. To date, no animal studies have examined changes in LTS bursts in the Mthal in PD models. To address this and to enable comparison of our data with previous reports of LTS bursts in reduced or anesthetized preparations, we searched for LTS-like events. Examples of events detected by these criteria are shown in Figure $4 A$. Overall, detected events occurred at very low rates, $\sim 1$ every $25 \mathrm{~s}$ (Fig. $4 E$ ), and mainly consisted of doublets ( $91.3 \%$ of 2380 events recorded in 181 neurons), with occasional triplets $(7.3 \%)$ or quadruplets $(1.4 \%)$.

The generation of LTS bursts requires de-inactivation of T-type calcium channels (Llinás and Jahnsen, 1982; Jahnsen and Llinás, 1984). To determine whether LTS-like events identified by electrophysiological criteria from our extracellular recordings depend on this channel, we infused the highly selective T-type calcium channel blocker NNC 55-0396 ([(1S,2S)-2-(2-(N-[(3-benzimidazol-2 yl)propyl]- $N$-methylamino) ethyl)-6-fluoro-1,2,3,4-tetrahydro-1-isopropyl-2-naphtyl cyclopropanecarboxylate dihydrochloride]; Tocris Bioscience) close to the recording site in the Mthal in 2 rats using an infusion cannula (Plastics One) glued to the side of the recording electrode bundle. Following established protocols, we infused $2.5 \mu \mathrm{l}$ of a $500 \mu \mathrm{m}$ solution (in $0.9 \%$ saline) at a rate of $0.5 \mu \mathrm{l} / \mathrm{min}$ using an automated syringe pump (IITC Life Sciences). In behavioral studies, this concentration of NNC 55-0396 has shown to be selective for T-type over high voltage activated channels (Tai et al., 2011). Recording quality was generally not affected, as indicated by consistent spike waveforms during drug infusion and before and after the insertion of the cannula. However, neurons were sometimes lost during cannula insertion or drug infusion and these recordings were not included in the analysis. At the end of the experiment, the microdrive was advanced $(\sim 35 \mu \mathrm{m})$ and no experiments were performed for $48 \mathrm{~h}$ to ensure clearance of the drug from the brain. For each rat, we performed only one drug infusion per week. In control experiments, $2.5 \mu \mathrm{l}$ of $0.9 \%$ saline was infused at the same rate.

As shown in Figure 4B, NNC 55-0396 infusion significantly reduced the incidence of LTS-like events $(1.30 \pm 0.23$ events. $10^{-2} / \mathrm{s}$ before vs $0.13 \pm 0.09$ events $\cdot 10^{-2} / \mathrm{s}$ after infusion, $n=8$, $p=0.014$, Wilcoxon paired $t$ test), suggesting that these detected events were dependent on T-type calcium channel activation. The overall firing rate was also significantly reduced $(1.74 \pm 0.35$ spikes/s before vs $0.44 \pm 0.15$ spikes/s after infusion, $n=8, p=$ 0.0078 , Wilcoxon paired $t$ test; Fig. $4 B$ ). Although not a major focus of the present study, this effect on overall firing rate raises the possibility that a proportion of single spikes recorded in Mthal may be the result of partial disinhibition of T-type calcium channel that fails to lead to a burst (Llinás and Steriade, 2006), in which case the true incidence of LTS-triggered events may be underestimated in freely moving animals. Control infusions of vehicle had no effect on LTS-like events or firing rates $(0.88 \pm$ 0.51 events $\cdot 10^{-2} / \mathrm{s}$ and $0.52 \pm 0.07$ spikes/s before infusion vs $0.69 \pm 0.4$ events $\cdot 10^{-2} / \mathrm{s}$ and $0.83 \pm 0.17$ spikes/s after infusion, $n=3$ ). Infusion of NNC 55-0396 did not affect the rate of high-frequency non-LTS-like bursts (same intraburst ISI criteria but no preburst ISI defined; $7.95 \pm 1.97$ bursts $\cdot 10^{-2} / \mathrm{s}$ before infusion vs $4.61 \pm 1.27$ bursts $\cdot 10^{-2} / \mathrm{s}$ after infusion, $n=8, p=$ 0.1610 , paired $t$ test; Fig. $4 B$ ) or the number of bursty neurons 




C


B
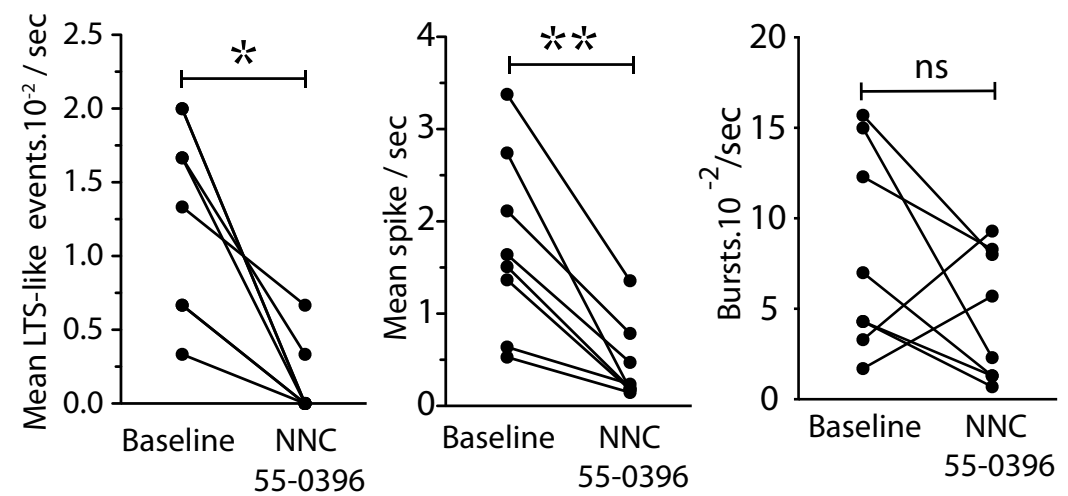

E

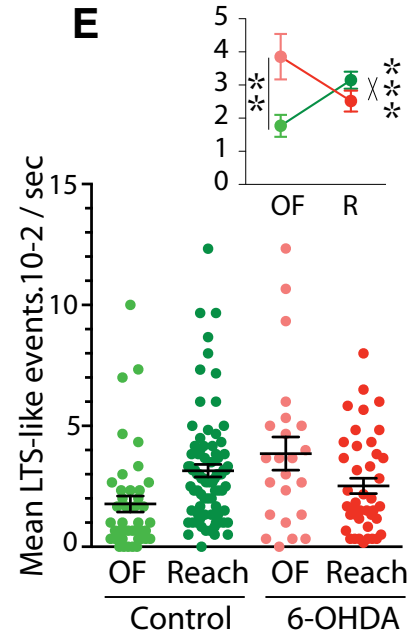

Figure 4. Effect of 6-OHDA lesion on LTS-like events in different behavioral tasks. A, Example LTS-like events with 2, 3, or 4 spikes (doublet, triplet, and quadruplet, respectively). $\boldsymbol{B}$, Mean occurrence of LTS-like events (left), firing rate (middle), and the rate of high-frequency but non-LTS-like, bursts (right) before and after intrathalamic infusion of NNC 55-0396, a T-type calcium channel blocker. Each line represents one neuron. ${ }^{* *} p<0.01 ;{ }^{*} p<0.05$. C, Relative frequency distributions of all ISIs from all spike trains that had high-frequency bursts (black line), preburst ISls from high-frequency bursts (gray line), and preburst ISIs from LTS-like events (green line) from control rats executing reaches. D, Mean \pm SEM of ISIs between spikes 1 and 2 (ISI \#1), 2 and 3 (ISI \#2), and 3 and 4 (ISI \#3) for each type of LTS-like event (doublets: open circles; triplets: dashed lines; quadruplets: solid lines). Data were pooled across open field and reaching behaviors and values were compared between control (green) and 6-OHDA (red) rats. $\boldsymbol{E}, \boldsymbol{F}$, Dots on scatter plots show mean occurrence of LTS-like events $(\boldsymbol{E})$ and percentage of all spikes in LTS-like events $(\boldsymbol{F})$ for each neuron recorded in control (green) and 6-OHDA (red) rats during open field (OF, light colors) and reaching (Reach, dark colors) tasks. Black lines represent grand mean \pm SEM over all neurons. Interaction plots (inset) show grand mean data comparing control and 6-OHDA lesioned groups during $\mathrm{OF}$ and reaching (R) behaviors (same color code as scatter plots). ${ }^{* *} p<0.01$ for group effect; ${ }^{* * *} p<$ 0.001 for interaction (cross); ${ }^{*} p<0.05$ for behavioral effect, 2 -factor ANOVAs with group (vertical statistics) and behavior (horizontal statistics) as factors.

assessed by the Kaneoke and Vitek (1996) method (7 neurons before, 4 neurons after, $p=0.282$, Fisher's exact test), but did affect reaching performance, with a small but significant decrease in the number of reaches $5-10 \mathrm{~min}$ after the infusion (before: $204 \pm 18$ reaches, after: $148 \pm 26$ reaches, $n=6$ sessions, $p=$ 0.0313 Wilcoxon matched-pairs signed-rank test). Nevertheless, rats did continue to make reaches after the infusion and, as detailed in the section LTS-like events, below, LTS-like events tended to decrease rather than increase in association with discrete reaching movements. Therefore, this $25 \%$ reduction in reaching is unlikely to account for all of the dramatic decrease in the incidence of LTS-like events, which fell to zero in the majority of recordings after the infusion.

To the best of our knowledge, this is the first report of highfrequency activity with features consistent with LTS-like bursts in freely moving control rodents; however, these LTS-like events are rare. To determine whether LTS-like events are indeed more likely to be preceded by a prolonged silent period, we plotted in Figure $4 C$ the distribution of preburst ISIs for all high-frequency bursts (i.e., we did not define the preburst ISI; Fig. 4C, gray line) and compared this with the distribution of all ISIs from all spike trains with high-frequency bursts (Fig. $4 C$, black line). This analysis revealed small, distinct peaks in the distribution of preburst ISIs, suggesting that, among these high-frequency events, particular preburst intervals were unusually common. We also examined the frequency distribution of preburst ISIs for only LTS-like events (Fig. $4 C$, green line). The peaks in preburst intervals for LTS-like events precisely match the timing of peaks in the overall distribution of preburst intervals (Fig. 4C, gray line), but have a larger relative frequency. Cumulatively, preburst intervals between 0.1 and $0.3 \mathrm{~s}$ account for $52 \%(3022 / 5812)$ of all ISIs preceding LTS-like events and, in awake animals, preburst intervals can be long, with many exceeding $0.3 \mathrm{~s}$. The proportion of preburst intervals in the 0.1-0.3 s range appears higher than the ratios that occurred by chance in comparable data from preburst ISIs for high-frequency bursts $(31 \%, 3249 / 10479$; Fig. $4 C$, gray line) and all ISIs (30\%, 114011/380036; Fig. 4C, black line). These results highlight that LTS-like events in awake conditions are present, but are rare among all recorded spikes, and that about half of the high-frequency spiking events in the Mthal do not meet the criteria of LTS bursts. 
A feature of presumed LTS-like events noted in some previous studies in mammalian thalamus is that, when the mean values for each successive ISI are plotted, there is a tendency for ISIs to increase within the burst, most prominently for later intervals in longer sequences; that is, spike frequency adaptation ( $\mathrm{Lu}$ et al., 1992; Lacey et al., 2007; Parr-Brownlie et al., 2009; Nakamura et al., 2014). This is in contrast to the fixed ISI seen during "high tonic discharge" events recorded in awake avian thalamus (Goldberg and Fee, 2012). This effect can be seen in our data in individual example bursts such as those shown in Figure 4A. Because of the criteria used to identify bursts in extracellular recordings, the range of possible values for the first interval is by definition smaller than for later intervals, so the best quantitative comparisons are between the second and later intervals; that is, in bursts of at least 4 spikes. Long bursts were very rare in the present dataset perhaps due to the disruptive effects of multiple active inputs in the awake state and, although there may have been a trend for an increase from ISI \#2 to \#3 in control rats in Figure $4 D$, this did not reach significance ( $p=0.3840$, two-factor ANOVA, with ISI position $(2,3)$ and group (control and 6-OHDA lesion) as factors). Similarly, there was no significant difference between these intervals in quadruplets from lesioned rats.

To determine whether the overall occurrence of LTS-like events varied with behavioral state or if it is affected by 6-OHDA lesion, we performed the same two-way ANOVA analyses as for general bursts with factors of group (control, lesioned) and behavior (open-field, reaching) on two measures, the rate of LTSlike events and the percentage of all spikes in these events. This revealed a significant interaction between behavior and group for rate $\left(F_{(1,182)}=12.78, p=0.0004\right.$, Fig. $\left.4 E\right)$. The interaction plot in Figure $4 E$ shows that this was because of differences between the groups in open field, but not during reaching. In the open field, the rate of LTS-like events was higher in 6-OHDA lesioned rats than in control animals ( $p=0.0016$, Bonferroni post hoc test). In contrast, there was no difference between the groups during reaching ( $p=0.328)$, reflecting a decrease compared with open field for lesioned rats, but an increase for control animals. This disjunction suggests that the rate of LTS-like events was not a simple function of firing rate because the firing rate tended to be higher during reaching in both groups (Fig. $3 B$ ).

Figure $4 F$ shows the equivalent analysis of the percentage of all spikes in LTS-like events. There was again a significant interaction $\left(F_{(1,177)}=15.07, p=0.0001\right)$. Here, the interaction plot and post hoc analyses showed that, although this measure was similar in both groups during open field, in control rats, there was a significantly larger proportion of spikes in LTS-like events during reaching compared with open field ( $p=0.0001$, Bonferroni multiple comparisons), whereas 6-OHDA rats showed no difference between behaviors ( $p=0.1305)$.

Finally, we investigated whether the occurrence of LTS-like events was dependent on the dorsoventral location of recorded neurons using data from the reaching task. Interestingly, we found a small but significant relationship in control animals $\left(r^{2}=0.10, p<0.0044\right.$, data not shown), with LTS-like events being more common in superficial recording sites; that is, in the CB territory of Mthal, but not in 6-OHDA lesioned animals $\left(r^{2}=\right.$ $0.003, p=0.6996)$.

Therefore, overall, although 6-OHDA lesions had rather consistent effects on firing rate and general bursting across the two behavioral conditions, the effect on LTS-like events was more complex. One interpretation of the data in Figure 4, $E$ and $F$, is that dopamine depletion prevents the increase of LTS-like events seen in control animals during reaching compared with during general open-field activity. This raises the possibility that LTSlike events may have a special role in reaching and that this physiological characteristic of Mthal activity is particularly affected by dopamine depletion.

\section{Modulation of Mthal activity during reaching in control and 6-OHDA animals}

To examine in more detail the relationship of Mthal activity in relation to specific movements and to determine the effect of unilateral dopamine depletion on this, we analyzed changes in activity during individual reaches performed with the prelesion dominant paw. For this, we constructed PETHs of firing rate and rate of LTS-like events aligned to the paw position close to the food to detect modulations associated with the end of the forelimb reaching movement and onset of distal movements for grasping the food (Whishaw and Pellis, 1990; Hyland and Jordan, 1997). We calculated PETHs over 2 time frames: a short timescale $( \pm 0.5 \mathrm{~s})$ to examine specific changes in activity over the time that muscle activity related to the extension phase of the reach movement, grasping, and retraction occur (Hyland and Jordan, 1997) and a broader scale ( $\pm 4 \mathrm{~s}$ ) to show the time periods before and after the reach movement.

\section{Firing rate}

Analysis of firing rate revealed that many neurons in Mthal showed distinct modulations around the end of the reach, which were typically complex with peak and trough components, as illustrated by the representative short timescale PETH from a neuron from a control rat in Figure 5A. The modulation pattern for all recorded Mthal neurons in control rats, including those with no obvious response, is shown in Figure $5 C$. The majority of Mthal neurons displayed a clear modulation in firing rate during the reaching task. The pattern was dominated by an inhibition after reach-end, but many neurons also showed a preceding excitation tightly aligned to the moment the paw broke the light beam 2 next to the food and some neurons also showed a distinct inhibition before the excitation.

Figure $6 \mathrm{~A}$ shows the grand mean average PETH for all recorded neurons in control animals over the same short timescale. This confirmed that, on average, Mthal output was characterized by three distinct phases of modulation around reach-end. Figure $6 \mathrm{C}$ shows the grand mean average over the longer timescale; this revealed additional broad excitatory and inhibitory modulations with an almost sinusoidal appearance before and after the discrete reach-end modulations. The broad excitation occurred over the time that rats were likely orienting to the feeder, whereas the suppression (with phasic components superimposed) occurred over the reach period itself and the postreach period that would have included food manipulation and consumption. From these group average data over the 2 timescales, we identified 2 peak $(\mathrm{P})$ and 3 trough (T) epochs for quantitative analysis (see Materials and Methods).

As illustrated by the example cell in Figure $5 B$, and at the population level by the group average PETH in Figure 6, $B$ and $D$, depletion of dopamine had a dramatic effect on the short and long timescale reach-related modulations of Mthal neurons. The group average plots revealed a profound diminution in Mthal responsiveness (Fig. $6 B, D$ ). The typical example neuron from a 6 -OHDA rat in Figure $5 B$ shows a central peak but no troughs around it. Overall, in 6-OHDA rats, few neurons showed multiple component responses and the T2 inhibition, which was very prominent in intact animals, was almost completely absent (Fig. 
$5 D)$. Over the wider timescale analysis, the grand mean plot (Fig. 6D) similarly showed a dramatic reduction in the later T3 inhibitory component, whereas the early excitation (P1) was present but appeared flatter than for control rats.

To compare statistically the amplitudes of response components in control and lesioned rats, we calculated the areas under the modulation curves for all neurons and compared epochs across groups using nonparametric Mann-Whitney U tests. The results of this analysis are shown in Figure $6 E$. For the peaks $\mathrm{P} 1$ and $\mathrm{P} 2$, there was no significant difference between control and lesioned animals. However, the analysis confirmed strong, selective effects on all the inhibitory components, with dopamine-depleted animals having reduced inhibitory troughs at $\mathrm{T} 1 \quad(p=$ 0.0351 ), and effectively completely abolished T2 and T3 $(p<0.0001)$.

\section{LTS-like events}

To determine whether these events were associated with a specific phase of reaching movements, we constructed grand mean PETH of LTS-like event occurrences aligned to the terminal part of the reach across all neurons and quantified the same peak (P1, P2) and trough (T1, T2, T3) epochs (Figs. 7A-D) as for firing rate. In control rats, the grand-mean average indicated modulation during some but not all of these epochs. Therefore, compared with baseline occurrence rate $(5.6 \pm 0.50$ LTS bursts $10^{-2} / \mathrm{s}$ ), there was a significant reduction over the P2 epoch $\left(4.2 \pm 0.51\right.$ events $\cdot 10^{-2} / \mathrm{s}, p=0.0005$, Friedman test with Dunn's multiple comparison) and the T2 epoch $(2.2 \pm 0.29$ events $\cdot 10^{-2} / \mathrm{s}, p<0.0001$; Fig. $7 A$ ). Inspection of the long timescale PETH in Figure $7 C$ suggested a similar sinusoidal pattern in LTS-like event occurrence as for firing rate, with an increased occurrence during the P1 epoch $(6.3 \pm 0.39$ events $\left.10^{-2} / \mathrm{s}, p=0.0343\right)$.

The most dramatic firing rate modulations over the short timescale analysis were between the $\mathrm{P} 2$ peak and T2 trough. Although there was no evidence of a P2 peak in LTS-like events incidence, to determine whether they tracked with the dramatic change in firing rate between these epochs, we calculated the change in LTS-like events and firing rate between P2 and T2 for each neuron and the results for control rats are shown in Figure 7, $E$ and $F$. Figure $7 E$ shows that significantly fewer LTS-like events occurred during T2 than P2 (respectively, $2.2 \pm 0.3$ events . $10^{-2} / \mathrm{s}$ vs $4.2 \pm 0.5$ events $\cdot 10^{-2} / \mathrm{s}, n=80, p<0.0001$, Wilcoxon test). Further examination of Figure $7 E$ shows that, at the individual neuron level, LTS-like events decreased during T2 compared with P2 in 37 neurons (46.3\%) and, for 13 of them, an event never occurred during T2. Only 12 neurons (15.0\%) showed the opposite relation, that LTS-like events were more common during T2 than P2; most of these $(n=8 / 12)$ showed LTS-like events only during T2. Six neurons (7.5\%) exhibited events with no obvious modulation in incidence across these epochs and 25 (31.2\%) had no detected events during these epochs. Regression
B 6-OHDA
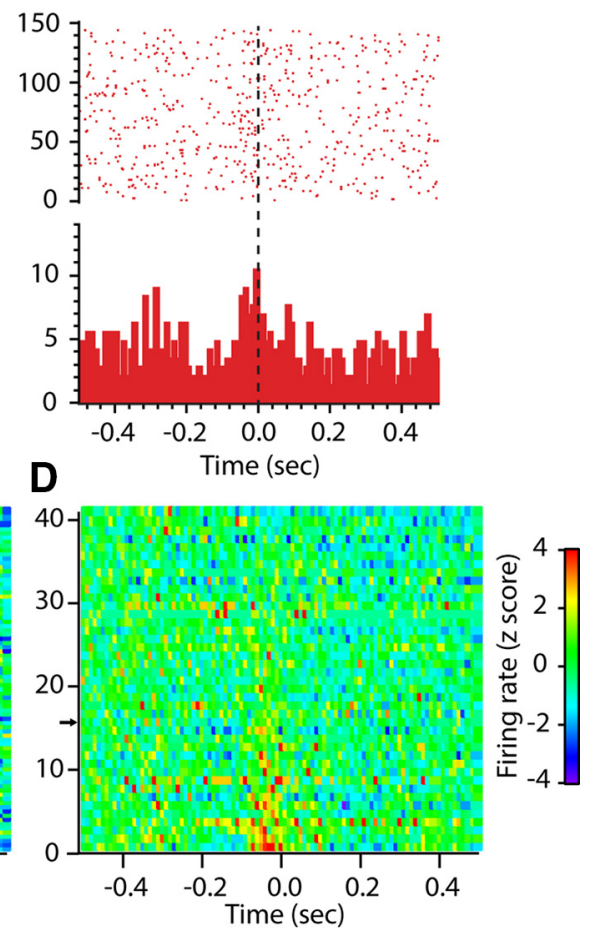

Figure 5. Modulation of spiking activity during reaching in control and 6-OHDA rats. $A, B$, Example PETHs of spiking activity aligned to the terminal part of the reach (interruption of beam 2: $t=0 \mathrm{~s}$ ) for one cell recorded in a control $(\boldsymbol{A}$, green) and 6-OHDA rats. On the raster plot (top), each line corresponds to one reaching trial and each dot shows when a spike occurred. $C, D$ firing rate and red indicating strong increases. Neurons were ordered with respect to their excitation peak (P2), with neuron \#1 displaying the strongest excitation. Example PETHs in $\boldsymbol{A}$ and $\boldsymbol{B}$ are indicated on the $y$-axis by a black arrow.

analysis of the difference $(\Delta)$ between the P2 and T2 periods for LTS-like events and firing rate (Fig. $7 F$ ) revealed a significant relationship $\left(r^{2}=0.12, p=0.0016\right)$, indicating that neurons with the strongest changes in LTS-like events also exhibited the strongest changes in firing rate. Together, these data confirm temporally precise modulation of LTS-like events during the reach.

The effect of dopamine depletion on reach-related occurrence of LTS-like events is shown in Figure 7, $B$ and $D$. These modulations in event occurrence during the reaching task seen in controls were completely lost in 6-OHDA animals over both short (Fig. 7B) and long (Fig. 7D) timescales. Therefore, the largest group of neurons $(20 / 41,48.8 \%)$ exhibited no change in LTS-like events between P2 and T2, 31.7\% exhibited a decrease in LTS-like events, and $19.5 \%$ exhibited an increase of these events between P2 and T2 (Fig. 7G). In addition, the regression equation of the change in LTS-like events with the change in firing rate did not differ significantly from 0 (Fig. $7 H$ ).

Figure $7 I$ shows the quantitative analysis of the amplitude of LTS-like event modulations calculated from the area under the modulation curve. This confirmed that dopamine depletion led to a significantly reduced level of LTS-like events during the P1 epoch compared with control animals ( $p=0.0264$, Mann-Whitney $U$ test) and also a significantly smaller reduction in LTS-like events during T2 ( $p=0.0133)$.

In summary, these data show that both firing rate and incidence of LTS-like events are modulated during the performance of the reaching task. These two measures tracked together in some respects (e.g., both measures reduced during T2), but dif- 
A
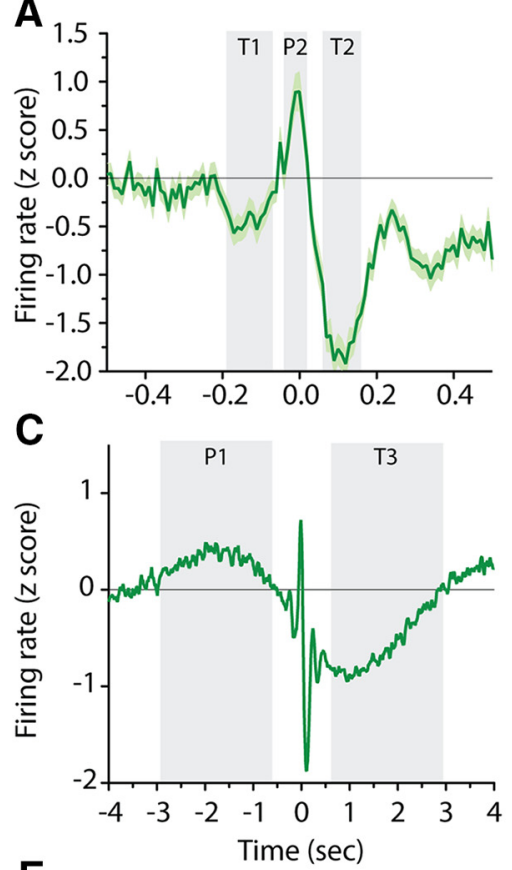

B

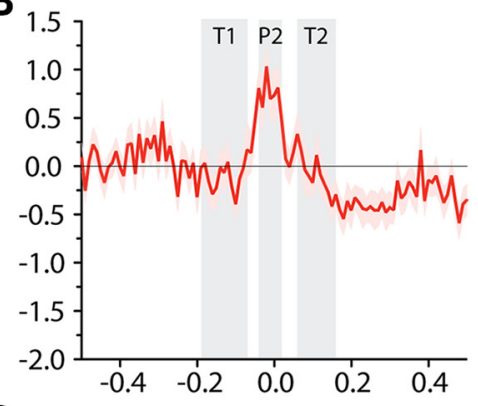

D

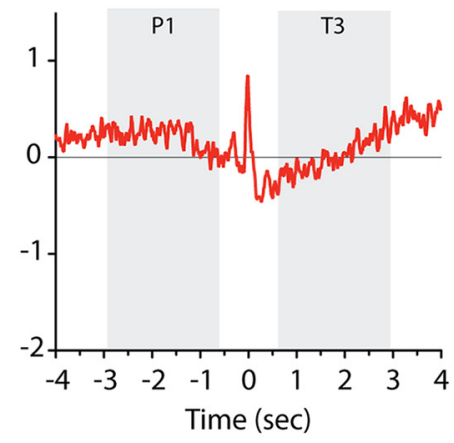

E
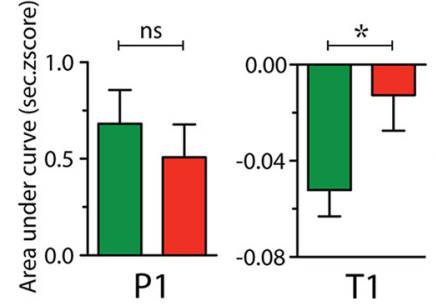

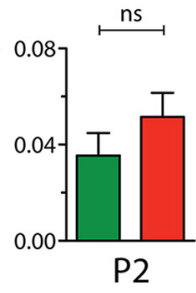

P2



T2

Figure 6. Reach-related modulations of spiking activity in 6-OHDA rats are characterized by absent troughs. $\boldsymbol{A}-\boldsymbol{D}$, Mean firing rate ( $z$-score) across all recorded neurons in control $(\boldsymbol{A}, \boldsymbol{C}$, green) and 6-OHDA $(\boldsymbol{B}, \boldsymbol{D}$, red) rats aligned to the terminal part of the reach (interruption of beam $2: t=0 \mathrm{~s}$ ) at narrow $( \pm 0.5 \mathrm{~s} ; \boldsymbol{A}, \boldsymbol{B}$, ) and wide timescales ( $\pm 4 \mathrm{~s} ; \boldsymbol{C}, \boldsymbol{D})$. P1-P2 and T1-T3 (shaded in light gray) show the 5 periods defined as peaks and troughs, respectively. In $\boldsymbol{A}$ and $\boldsymbol{B}$, results are presented as mean (thick line) \pm SEM (shaded area). In $\boldsymbol{C}$ and $\boldsymbol{D}$, results are presented as the smoothed mean. $\boldsymbol{E}$, Area under curve for P1-P2 and T1-T3 periods indicate the amplitude of the modulations for control (green) and 6-0HDA (red) rats. ${ }^{*} p<0.05$; ${ }^{* * *} p<0.0001$, Mann-Whitney tests.

fered at the moment the paw broke the light beam at the end of the reach, where there was a peak in firing rate but not in LTS-like event activity. Importantly, in dopamine-depleted animals, the inhibitory firing rate and LTS-like event modulations were both severely compromised, but the excitatory firing rate peak was not affected. These data suggest a particularly important role for normal dopamine innervation of BG in enabling timed inhibitory events in Mthal during skilled discrete limb movements, whereas excitatory events appear to be less affected.

\section{Correlation of reaching-related activity and location of Mthal neuron}

To determine whether the different patterns of reach-related spiking activity in Mthal neurons shown in Figure 5 were dependent on the location of neurons, we plotted each neuron's activity during the 5 modulation epochs against its anteroposterior, mediolateral, and dorsoventral coordinates. We did not find any relationship between the anteroposterior or mediolateral coordinates and modulation of firing rate during any epoch (data not shown). However, for control animals, regression analyses revealed a significant effect of dorsoventral location during some of the epochs (Fig. 8). These gradients in amplitude of

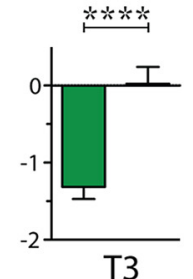

T3 different response components could reflect gradients in intensity of innervation from different inhibitory (BG) and excitatory sources ( $\mathrm{CB}$ and cortex).

For firing rate, the broad early peak (P1) in the long timescale analysis (Fig. $8 A$ ) became increasingly prominent in more ventral neurons, likely to be in the BG territory of Mthal $\left(r^{2}=0.12, p=\right.$ 0.0019). The first phasic inhibition (T1) also became more prominent (i.e., more negative $z$-score) the more ventral the neuron (Fig. $8 B ; r^{2}=0 / 10, p=0.0048$ ). In contrast, the analysis revealed that the phasic peak P2 (Fig. $8 C$ ) was larger in more dorsal neurons $\left(r^{2}=0.14, p=\right.$ 0.0008), which are likely to be in the $\mathrm{CB}$ territory of Mthal. Neither of the later inhibitory epochs (T2, T3) showed any significant relation to recording depth (Fig. $8 D, E)$. To assess modulation of LTS-like events during reaching over the same epochs as for firing rate, we analyzed the difference in LTS-like events between the P2 and T2 periods. As shown in Figure $8 F$, this measure was significantly related to the dorsoventral location of neurons in the Mthal $\left(r^{2}=0.08, p=0.0145\right)$. Neurons located more dorsally, in the CB territory, exhibited a decrease in LTS-like events from P2 to T2, whereas neurons situated more ventrally in the BG territory exhibited little change in LTS-like events between the two epochs.

In striking contrast to the control animal data, in 6-OHDA lesioned rats, none of the short timescale activity modulations (P2, T1, T2) showed any relation to neuron location (Fig. $8 H-J, L$ ). However, for long timescale epoch $\mathrm{P} 1$, the same relationship was seen as in control animals $\left(r^{2}=0.28, p=0.0004\right.$; Fig. $\left.8 G\right)$, whereas for T3 (Fig. $8 K$ ), recording depth showed a significant effect $\left(r^{2}=0.22, p=0.0019\right)$ that did not reach significance in controls.

In summary, Mthal neurons exhibited reaching-related activity that differs depending on their location. The early excitatory modulation (P1) became more prominent ventrally in the BG receiving $\mathrm{Mthal}$, whereas the excitation temporally aligned to reach end (P2) was more prominent dorsally in the CB territory. For inhibitions, $\mathrm{T} 1$ became more prominent ventrally. These gradations are consistent with the possibility that some components of firing rate modulation in Mthal are more regulated by BG inputs and others, along with occurrence of LTS-like events, by $\mathrm{CB}$ inputs. Dopamine depletion led to loss of these relationships for short timescale epochs.

\section{Discussion}

The main results of this study are summarized in Figure 9. We found that Mthal neurons show a distinct pattern of inhibitory and excitatory modulations in firing rate during a skilled reaching movement, with some evidence for regional variation that may reflect location in either CB- or BG-receiving territories. LTS-like events were rare, but their incidence was also mo- 
dulated during reaching. Finally, the reaching-related modulations in activity were significantly altered in chronic dopamine-depleted rats.

\section{Mthal neuronal activity is precisely modulated during skilled reaching movement}

We aligned PETHs to detect Mthal modulations associated with reach-end and grasping the food (Whishaw and Pellis, 1990; Hyland and Jordan, 1997) in a skilled-forelimb reaching task, with important homology in performance between rats and humans (Whishaw et al., 1992; Sacrey et al., 2009). Individual neurons showed different activity patterns, but a remarkably consistent sequence occurred across the whole population. Over short timescales relative to reach-end, there was a triphasic inhibition-excitation-inhibition sequence. Neurons that did not show all phases still had components that aligned temporally with those seen in the full sequence. It is likely that the temporally precise activity pattern we observed in Mthal neurons relates to the highly organized and complex sequence of muscle activation during reaching movements (Whishaw et al., 1994; Hyland and Jordan, 1997).

Strong modulations are also seen in monkey Mthal during accurate reaching movements, including excitations, inhibitions (Strick, 1976; Inase et al., 1996), and biphasic excitation-inhibition sequences (Anderson and Turner, 1991). These studies did not report prominent triphasic patterns, perhaps because there was no grasp component and data were centered on reach onset. Sequences of inhibition and excitation have also been observed in homologous regions in birds during the generation of highly structured, coordinated song (Goldberg and Fee, 2012). Interestingly, complex inhibition-excitation-inhibition sequences in Mthal are predicted by considering the temporally dispersed interactions through hyperdirect, direct, and indirect BG pathways and are proposed to be important for accurate selection, initiation, and termination of specific movements (Nambu, 2004).

Within Mthal, there is complex regional variation in afferent inputs, with relative separation of CB- and BGreceiving territories, particularly evident in monkey and cat (Anderson and DeVito, 1987; Anderson and Turner, 1991; Sakai et al., 1996), but also discernable in rats (Kuramoto et al., 2011; Nakamura et al., 2014). This complex innervation pattern may lead to different neuronal response modulations across Mthal. Indeed, we found variations in movement-related activity depending on the location of recorded neurons. In particular, a phasic inhibitory component that occurred just before the grasp-

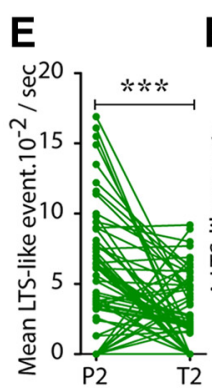

\section{A Control}
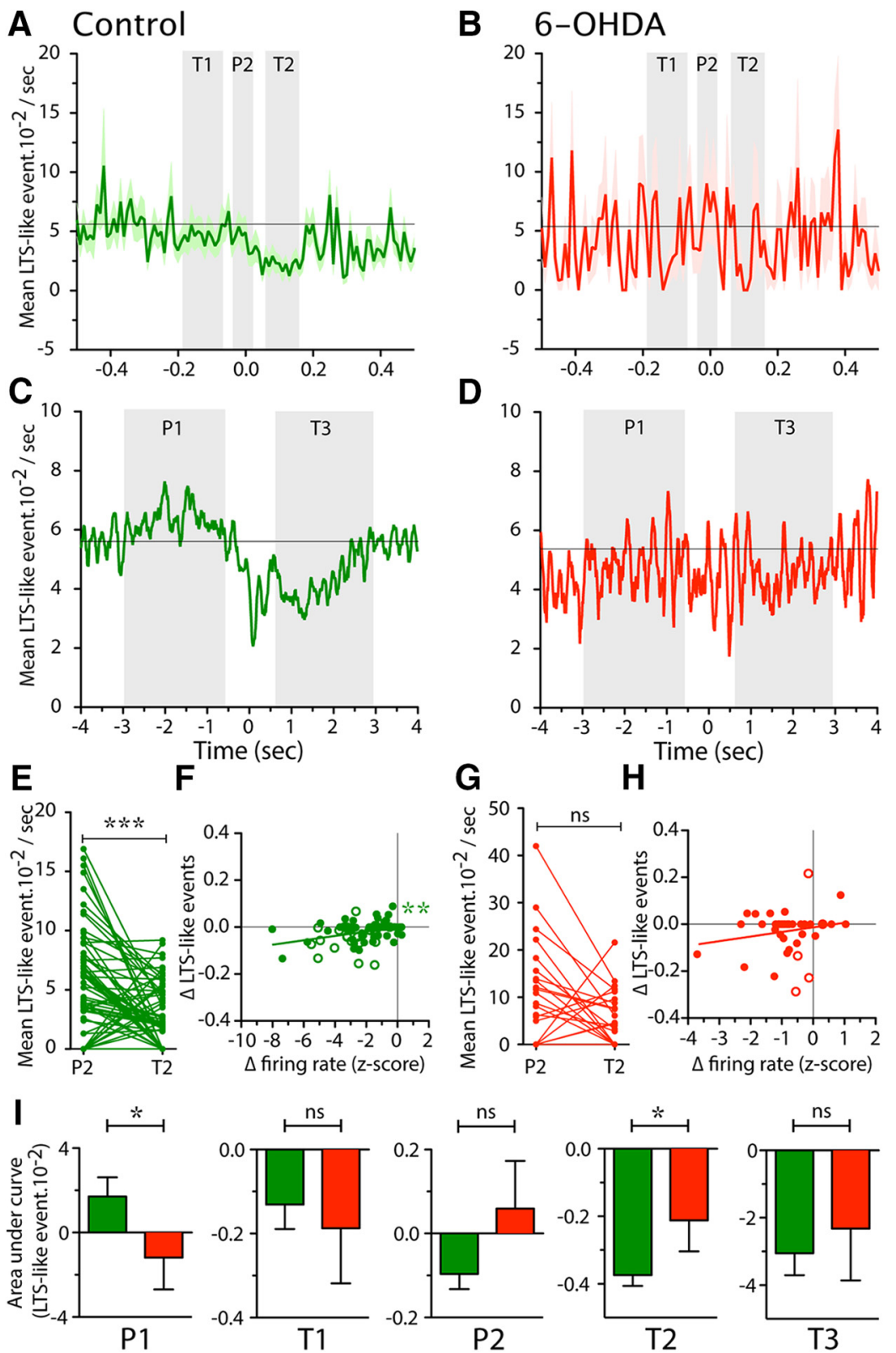

$\mathrm{H}$
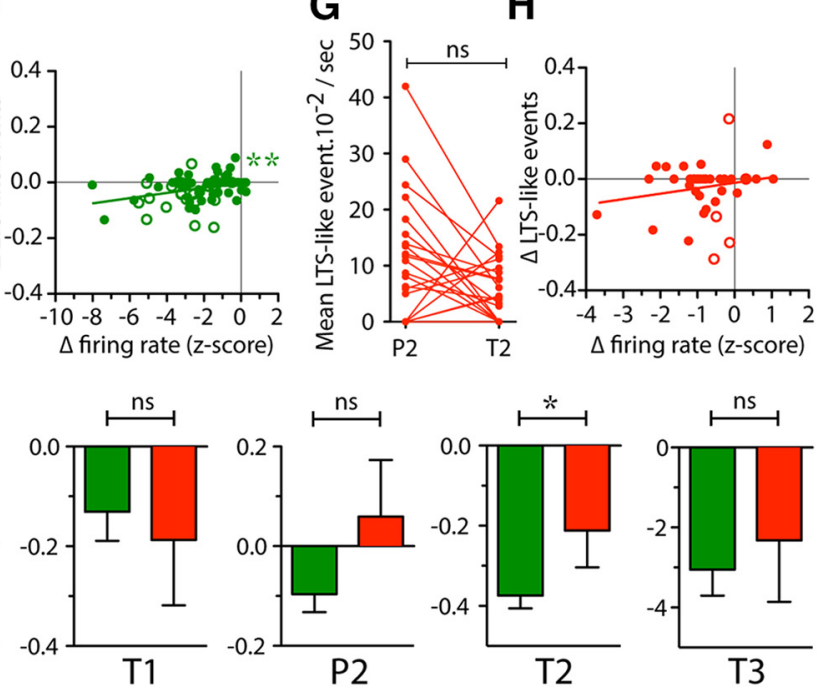

Figure 7. Modulation of LTS-like events during reaching in control and 6-OHDA lesioned rats. $\boldsymbol{A}-\boldsymbol{D}$, Mean occurrence of LTS-like events across all recorded neurons in control $(\boldsymbol{A}, \boldsymbol{C}$, green) and 6-0HDA $(\boldsymbol{B}, \boldsymbol{D}$, red) rats, aligned to the terminal part of the reach (interruption of beam $2: t=0 s$ ) during narrow $( \pm 0.5 s ; \boldsymbol{A}, \boldsymbol{B})$ and wide timescales $( \pm 4 \mathrm{~s} ; \boldsymbol{C}, \boldsymbol{D})$. P1-P2 and T1-T3 (shaded in light gray) show the 5 periods we defined as peaks and troughs, respectively, consistent with Figure $6 . \ln \boldsymbol{A}$ and $\boldsymbol{B}$, data are presented as mean (thick line) \pm SEM (shaded area) and in $\boldsymbol{C}$ and $\boldsymbol{D}$ as the smoothed mean. $\boldsymbol{E}, \boldsymbol{G}$, Plot of the mean occurrence of LTS-like events at the time of $\mathrm{P} 2$ and $\mathrm{T} 2$ for each neuron recorded in control $\left(\boldsymbol{E}\right.$, green) and 6-0HDA (G, red) rats. ${ }^{* *} p<0.001$, paired $t$ test. $\boldsymbol{F}, \boldsymbol{H}$, Correlation plots of the change $(\Delta)$ in LTS-like events (difference between LTS-like events at P2 and T2) with the $\Delta$ in firing rate (difference of mean spiking activity at P2 and T2). Each dot represents one neuron, filled circles are neurons recorded in BG territory (control rats: G, green; 6-0HDA rats: I, red), open circles represent neurons recorded in (B territory of Mthal. Straight lines represent the linear regression. ${ }^{* *}$ Linear regression significantly different from $0(p<0.01)$. $I$, Mean area under modulation curves for P1-P2 and T1-T3 periods for control (green) and 6-0HDA (red) rats. ${ }^{*} p<0.05$; Mann-Whitney tests.

ing phase was more prominent at deeper locations in the BG territory, whereas later inhibitory components did not change with depth. In contrast, the phasic peak in firing rate at reach-end was more prominent in dorsal regions, likely dominated by $\mathrm{CB}$ input, which is consistent for a role for glutamatergic $\mathrm{CB}$ pathways. Finally, Mthal CB and BG territories receive excitatory input from different areas of cerebral cortex, with the CB territory dominated by motor cortex and the BG territory by premotor cortex projections. Therefore, the significant increase in very 

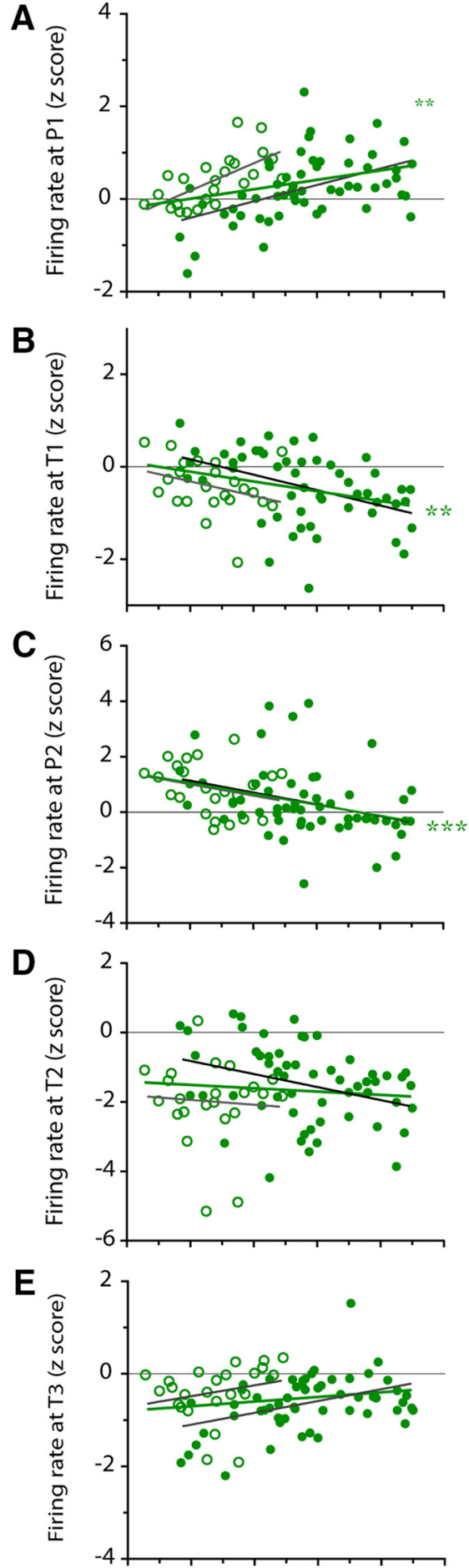

$\mathbf{F}$

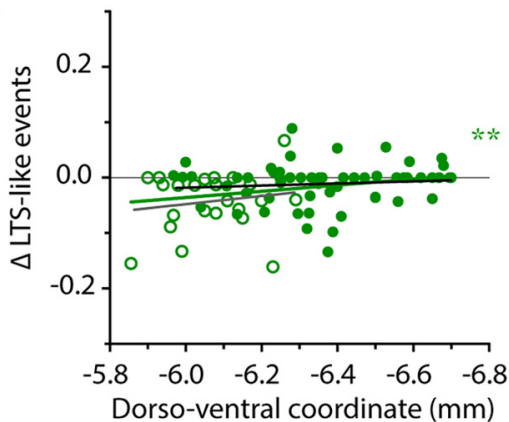

G

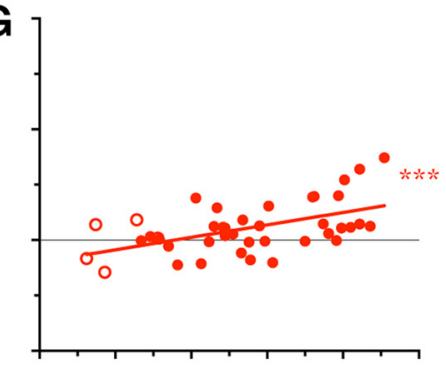

H
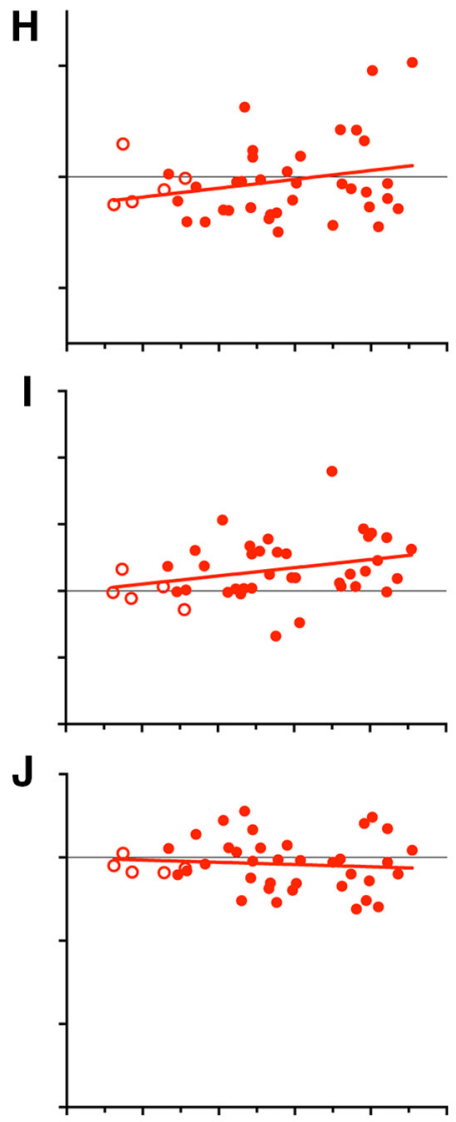

K

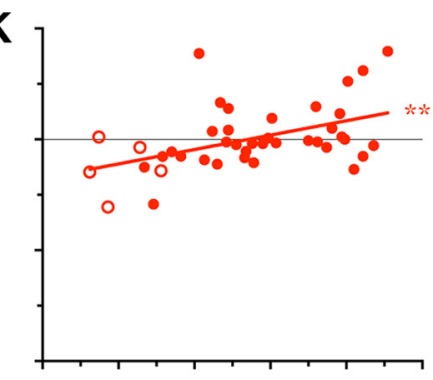

L

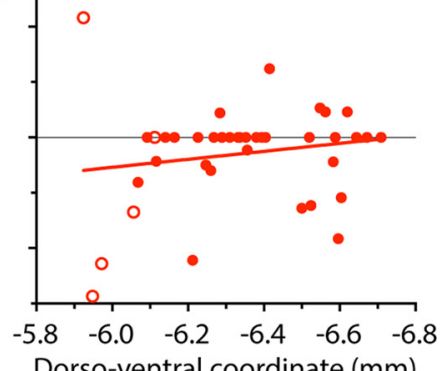

early excitatory activity in ventral compared with dorsal recordings could reflect the stronger influence of premotor cortex and SMA activity associated with movement preparation (Weinrich and Wise, 1982; Weinrich et al., 1984; Tanji et al., 1988; Crutcher and Alexander, 1990; Alexander and Crutcher, 1990b, c).

In addition to modulations of overall rate, burst-firing patterns are important for determining functional impact of neural activity. High-frequency LTS bursts in Mthal in mammals are assumed to increase synaptic reliability (Lisman, 1997; Bosch-Bouju et al., 2013) and so could powerfully modify activity in downstream structures. We identified events that met previously reported electrophysiological criteria for isolating LTS-like events. Our data highlight that high-frequency spiking can occur for short periods in response to movement execution, so it is important to carefully distinguish high-frequency bursts from LTS-like events. Overall, the rate of these LTS-like events in behaving rats was very low in our study and, although similar rates have been observed in the Mthal when the cortex had been activated in anesthetized rats (Nakamura et al., 2014), that study also reported a much higher rate of LTS bursts $(80 \%$ of spikes in LTS bursts and $\sim 1$ LTS burst/s) during slow-wave activity associated with deep anesthesia, consistent with the idea that LTS bursts are common during periods of inattention (Ramcharan et al., 2005). This state-dependent effect of LTSlike events may reflect less synchronous GABAergic BG inputs to Mthal in the awake (or lightly anesthetized) state and/or enhanced excitatory afferent input, both of which would reduce chances for LTS burst initiation.

The percentage of spikes in LTS-like events was significantly higher when control animals were performing discrete reaches than during open-field behavior;

\footnotetext{
Figure 8. Movement-related activity depends on the neuron's location in the Mthal. Scattergrams of the dorsoventral location of recorded neurons plotted against their firing rate at the P1 $(\boldsymbol{A}, \boldsymbol{G}), \mathrm{T} 1(\boldsymbol{B}, \boldsymbol{H}), \mathrm{P} 2(\boldsymbol{C}, \boldsymbol{I}), \mathrm{T} 2(\boldsymbol{D}, \boldsymbol{J}), \mathrm{T} 3(\boldsymbol{E}, \boldsymbol{K})$ and $\Delta$ occurrence of $L T S$-like events $(\boldsymbol{F}, \boldsymbol{L})$. For all graphs, filled circles represent neurons recorded in $B G$ territory and open circles represent neurons recorded in $C B$ territory of Mthal. Data for control and 6-0HDA rats are represented in green $(\boldsymbol{A}-\boldsymbol{F})$ and red $(\mathbf{G}-\boldsymbol{L})$, respectively. Linear regressions are represented by straight lines (green for all neurons in control rats, light gray for CB territory control neurons, dark gray for BG territory control neurons, red for 6-OHDA lesioned rats) and asterisks indicate when the regression was significantly different from 0 $\left({ }^{* *} p<0.01 ;{ }^{* * *} p<0.001\right)$.
} 
however, there was a significant reduction of LTS-like events at the time of reach-end. Therefore, discrete timelocked increases in efficacy of synaptic transmission through enhanced incidence of particular high-frequency bursts does not appear necessary for generation of discrete movements. The possible functional roles of these contrasting effects at different timescales thus remain enigmatic.

\section{Mthal neuronal activity is selectively impaired in 6-OHDA rats}

Neuronal activity in 6-OHDA rats revealed important differences that may underlie dysfunctional movements in PD. We found increased mean firing rates across open-field and reaching behaviors in lesioned animals. This contrasts with early theories of circuit dysfunction that assumed that hyperactivity in inhibitory BG output in PD would reduce Mthal neuronal activity (Albin et al., 1989; Alexander and Crutcher, 1990a; DeLong, 1990), supported by some early studies in parkinsonian animals (Voloshin et al., 1994; Schneider and Rothblat, 1996). However, more recent studies did not find the predicted decreases (Pessiglione et al., 2005; Lobb et al., 2013). Together, these data suggest that, in behaving animals, a reduction in overall Mthal firing rate is not a necessary consequence of dopamine depletion.

More recently, increased oscillatory and bursty firing patterns in BG and cortex after dopamine depletion have been identified as key pathological features of PD (Bergman et al., 1994, 1998; Wichmann and DeLong, 1996; Boraud et al., 2002; Goldberg et al., 2002; Walters et al., 2007; Galvan and Wichmann, 2008; Pasquereau and Turner, 2011). PD patients also show presumed pathological neuronal activity in the tremor range (Lenz et al., 1995; Garonzik et al., 2002). In contrast, we saw nonsignificant effects of dopamine depletion on measures of general bursting in Mthal, except for a reduction in the number of bursty neurons. This difference may be because PD model rats do not display limb tremor. The role of these pathological signals in Mthal is complex because, although VL thalamotomy and deep brain stimulation appear to treat tremor, their role in akinesia and fine motor control is unclear (Perret, 1968, 1970; Okun and Vitek, 2004; Chen et al., 2006; Duval et al., 2006).

Previous studies predicted that PD pathophysiology may include increases in LTS bursts (Paré et al., 1990; Jeanmonod et al., 1996; Magnin et al., 2000; Guo et al., 2008). In contrast, we found only a subtle effect of dopamine depletion on LTSlike events and this appeared opposite to that proposed; whereas normal rats showed an increase in such events when engaged in the reaching task, lesioned rats failed to show the increase. Therefore, LTS-like events in control conditions may have a role when switching to a discrete movement mode and, when this fails in dopamine-depleted animals, task performance is also impaired.
The most dramatic finding of the present study came from our ability to compare activity during those reaches that were executed by parkinsonian animals to reveal pathologies that may be associated with movement performance rather than just with akinesia. First, disruption of firing rate modulation early in the reach ( $\mathrm{P} 1$ epoch) may correlate to known impairments in posture and compensatory adjustments observed in 6-OHDA lesioned rats and PD patients (Miklyaeva et al., 1994; Whishaw et al., 2002). In contrast, we found the phasic peak at reach-end (P2) was not altered, suggesting that it reflects activity in excitatory afferents that are not affected directly by dopamine depletion, for example, from the CB. Second, around the time of reach-end, lesioned rats did not show the movement-related troughs in spiking activity that gave rise to the normal triphasic pattern and troughs in LTS-like events were also abolished. Given that BG inputs are GABAergic, loss of firing-rate troughs would be consistent with a specific deficit in BG inputs to Mthal. These dramatic changes were not associated with alterations in velocity of reach-end, but instead may relate to disordered fine control of the digits, which were described previously using high-speed video (Miklyaeva et al., 1994; Whishaw et al., 2002). We also found that there were subtle differences in modulations between Mthal territories, but these changes require further investigation before they can be fully understood. Importantly, the loss of particular components of Mthal activity normally associated with reach-end may provide a 
neurophysiological basis for the impairments seen in the fine motor control of digits for food grasping in parkinsonian patients and rats.

\section{References}

Albin RL, Young AB, Penney JB (1989) The functional anatomy of basal ganglia disorders. Trends Neurosci 12:366-375. CrossRef Medline

Alexander GE, Crutcher MD (1990a) Functional architecture of basal ganglia circuits: neural substrates of parallel processing. Trends Neurosci 13:266-271. CrossRef Medline

Alexander GE, Crutcher MD (1990b) Neural representations of the target (goal) of visually guided arm movements in three motor areas of the monkey. J Neurophysiol 64:164-178. Medline

Alexander GE, Crutcher MD (1990c) Preparation for movement: neural representations of intended direction in three motor areas of the monkey. J Neurophysiol 64:133-150. Medline

Anderson ME, DeVito JL (1987) An analysis of potentially converging inputs to the rostral ventral thalamic nuclei of the cat. Exp Brain Res 68: 260-276. Medline

Anderson ME, Turner RS (1991) Activity of neurons in cerebellar-receiving and pallidal-receiving areas of the thalamus of the behaving monkey. J Neurophysiol 66:879-893. Medline

Bergman H, Wichmann T, Karmon B, DeLong MR (1994) The primate subthalamic nucleus. II. Neuronal activity in the MPTP model of parkinsonism. J Neurophysiol 72:507-520. Medline

Bergman H, Feingold A, Nini A, Raz A, Slovin H, Abeles M, Vaadia E (1998) Physiological aspects of information processing in the basal ganglia of normal and parkinsonian primates. Trends Neurosci 21:32-38. CrossRef Medline

Boraud T, Bezard E, Bioulac B, Gross CE (2002) From single extracellular unit recording in experimental and human Parkinsonism to the development of a functional concept of the role played by the basal ganglia in motor control. Prog Neurobiol 66:265-283. CrossRef Medline

Bosch-Bouju C, Hyland BI, Parr-Brownlie LC (2013) Motor thalamus integration of cortical, cerebellar and basal ganglia information: implications for normal and parkinsonian conditions. Front Comput Neurosci 7:163. CrossRef Medline

Butler EG, Horne MK, Hawkins NJ (1992) The activity of monkey thalamic and motor cortical neurones in a skilled, ballistic movement. J Physiol 445:25-48. Medline

Butler EG, Finkelstein DI, Harvey MC, Churchward PR, Forlano LM, Horne MK (1996) The relationship between monkey ventrolateral thalamic nucleus activity and kinematic parameters of wrist movement. Brain Res 736:146-159. CrossRef Medline

Chen H, Hua SE, Smith MA, Lenz FA, Shadmehr R (2006) Effects of human cerebellar thalamus disruptive on adaptive control of reaching. Cereb Cortex 16:1462-1473. CrossRef Medline

Crutcher MD, Alexander GE (1990) Movement-related neuronal activity selectively coding either direction or muscle pattern in three motor areas of the monkey. J Neurophysiol 64:151-163. Medline

DeLong MR (1990) Primate models of movement disorders of basal ganglia origin. Trends Neurosci 13:281-285. CrossRef Medline

Denny-Brown D (1968) Symposium on diseases of the basal ganglia: nature of dystonia. I. Rev Bras Med 25:769-773. Medline

Duval C, Panisset M, Strafella AP, Sadikot AF (2006) The impact of ventrolateral thalamotomy on tremor and voluntary motor behavior in patients with Parkinson's disease. Exp Brain Res 170:160-171. CrossRef Medline

Ferguson JE, Boldt C, Redish AD (2009) Creating low-impedance tetrodes by electroplating with additives. Sens Actuators A Phys 156:388-393. CrossRef Medline

Forlano LM, Horne MK, Butler EG, Finkelstein D (1993) Neural activity in the monkey anterior ventrolateral thalamus during trained, ballistic movements. J Neurophysiol 70:2276-2288. Medline

Galvan A, Wichmann T (2008) Pathophysiology of parkinsonism. Clin Neurophysiol 119:1459-1474. CrossRef Medline

Garonzik IM, Hua SE, Ohara S, Lenz FA (2002) Intraoperative microelectrode and semi-microelectrode recording during the physiological localization of the thalamic nucleus ventral intermediate. Mov Disord 17: S135-S144. CrossRef Medline

Glickstein M, Stein J (1991) Paradoxical movement in Parkinson's disease. Trends Neurosci 14:480-482. CrossRef Medline

Goldberg JA, Boraud T, Maraton S, Haber SN, Vaadia E, Bergman H (2002) Enhanced synchrony among primary motor cortex neurons in the 1-methyl-4-phenyl-1,2,3,6-tetrahydropyridine primate model of Parkinson's disease. J Neurosci 22:4639-4653. Medline

Goldberg JH, Fee MS (2012) A cortical motor nucleus drives the basal ganglia-recipient thalamus in singing birds. Nat Neurosci 15:620-627. CrossRef Medline

Guo Y, Rubin JE, McIntyre CC, Vitek JL, Terman D (2008) Thalamocortical relay fidelity varies across subthalamic nucleus deep brain stimulation protocols in a data-driven computational model. J Neurophysiol 99: 1477-1492. CrossRef Medline

Horne MK, Porter R (1980) The discharges during movement of cells in the ventrolateral thalamus of the conscious monkey. J Physiol 304:349-372. Medline

Hyland BI, Jordan VM (1997) Muscle activity during forelimb reaching movements in rats. Behav Brain Res 85:175-186. CrossRef Medline

Inase M, Buford JA, Anderson ME (1996) Changes in the control of arm position, movement, and thalamic discharge during local inactivation in the globus pallidus of the monkey. J Neurophysiol 75:1087-1104. Medline

Ivanusic JJ, Bourke DW, Xu ZM, Butler EG, Horne MK (2005) Cerebellar thalamic activity in the macaque monkey encodes the duration but not the force or velocity of wrist movement. Brain Res 1041:181-197. CrossRef Medline

Jahnsen H, Llinás R (1984) Ionic basis for the electro-responsiveness and oscillatory properties of guinea-pig thalamic neurones in vitro. J Physiol 349:227-247. Medline

Jeanmonod D, Magnin M, Morel A (1996) Low-threshold calcium spike bursts in the human thalamus: common physiopathology for sensory, motor and limbic positive symptoms. Brain 119:363-375. CrossRef Medline

Kaneoke Y, Vitek JL (1996) Burst and oscillation as disparate neuronal properties. J Neurosci Methods 68:211-223. CrossRef Medline

Kojima S, Doupe AJ (2009) Activity propagation in an avian basal gangliathalamocortical circuit essential for vocal learning. J Neurosci 29:47824793. CrossRef Medline

Kunimatsu J, Tanaka M (2010) Roles of the primate motor thalamus in the generation of antisaccades. J Neurosci 30:5108-5117. CrossRef Medline

Kuramoto E, Fujiyama F, Nakamura KC, Tanaka Y, Hioki H, Kaneko T (2011) Complementary distribution of glutamatergic cerebellar and GABAergic basal ganglia afferents to the rat motor thalamic nuclei. Eur J Neurosci 33:95-109. CrossRef Medline

Kurata K (2005) Activity properties and location of neurons in the motor thalamus that project to the cortical motor areas in monkeys. J Neurophysiol 94:550-566. CrossRef Medline

Lacey CJ, Bolam JP, Magill PJ (2007) Novel and distinct operational principles of intralaminar thalamic neurons and their striatal projections. J Neurosci 27:4374-4384. CrossRef Medline

Leblois A, Bodor AL, Person AL, Perkel DJ (2009) Millisecond timescale disinhibition mediates fast information transmission through an avian basal ganglia loop. J Neurosci 29:15420-15433. CrossRef Medline

Lenz FA, Normand SL, Kwan HC, Andrews D, Rowland LH, Jones MW, Seike M, Lin YC, Tasker RR, Dostrovsky JO (1995) Statistical prediction of the optimal site for thalamotomy in parkinsonian tremor. Mov Disord 10: 318-328. CrossRef Medline

Lisman JE (1997) Bursts as a unit of neural information: making unreliable synapses reliable. Trends Neurosci 20:38-43. CrossRef Medline

Llinás R, Jahnsen H (1982) Electrophysiology of mammalian thalamic neurones in vitro. Nature 297:406-408. CrossRef Medline

Llinás RR, Steriade M (2006) Bursting of thalamic neurons and states of vigilance. J Neurophysiol 95:3297-3308. CrossRef Medline

Lobb CJ, Zaheer AK, Smith Y, Jaeger D (2013) In vivo electrophysiology of nigral and thalamic neurons in alpha-synuclein-overexpressing mice highlights differences from toxin-based models of parkinsonism. J Neurophysiol 110:2792-2805. CrossRef Medline

Lu SM, Guido W, Sherman SM (1992) Effects of membrane voltage on receptive field properties of lateral geniculate neurons in the cat: contributions of the low-threshold Ca2 + conductance. J Neurophysiol 68: 2185-2198. Medline

MacPherson JM, Rasmusson DD, Murphy JT (1980) Activities of neurons in "motor" thalamus during control of limb movement in the primate. J Neurophysiol 44:11-28. Medline

Magnin M, Morel A, Jeanmonod D (2000) Single-unit analysis of the palli- 
dum, thalamus and subthalamic nucleus in parkinsonian patients. Neuroscience 96:549-564. CrossRef Medline

Middleton FA, Strick PL (2000) Basal ganglia and cerebellar loops: motor and cognitive circuits. Brain Res Rev 31:236-250. CrossRef Medline

Miklyaeva EI, Castañeda E, Whishaw IQ (1994) Skilled reaching deficits in unilateral dopamine-depleted rats: impairments in movement and posture and compensatory adjustments. J Neurosci 14:7148-7158. Medline

Nakamura KC, Sharott A, Magill PJ (2014) Temporal coupling with cortex distinguishes spontaneous neuronal activities in identified basal gangliarecipient and cerebellar-recipient zones of the motor thalamus. Cereb Cortex 24:81-97. CrossRef Medline

Nambu A (2004) A new dynamic model of the cortico-basal ganglia loop. Prog Brain Res 143:461-466. CrossRef Medline

Nambu A, Yoshida S, Jinnai K (1991) Movement-related activity of thalamic neurons with input from the globus pallidus and projection to the motor cortex in the monkey. Exp Brain Res 84:279-284. Medline

Obeso JA, Rodríguez-Oroz MC, Rodríguez M, Lanciego JL, Artieda J, Gonzalo N, Olanow CW (2000) Pathophysiology of the basal ganglia in Parkinson's disease. Trends Neurosci 23:S8-S19. CrossRef Medline

Okun MS, Vitek JL (2004) Lesion therapy for Parkinson's disease and other Mov Disord: update and controversies. Mov Disord 19:375-389. CrossRef Medline

Olsson M, Nikkhah G, Bentlage C, Björklund A (1995) Forelimb akinesia in the rat Parkinson model: differential effects of dopamine agonists and nigral transplants as assessed by a new stepping test. J Neurosci 15:38633875. Medline

Paré D, Curro'Dossi R, Steriade M (1990) Neuronal basis of the parkinsonian resting tremor: a hypothesis and its implications for treatment. Neuroscience 35:217-226. CrossRef Medline

Parr-Brownlie LC, Hyland BI (2005) Bradykinesia induced by dopamine D2 receptor blockade is associated with reduced motor cortex activity in the rat. J Neurosci 25:5700-5709. CrossRef Medline

Parr-Brownlie LC, Poloskey SL, Flanagan KK, Eisenhofer G, Bergstrom DA, Walters JR (2007) Dopamine lesion-induced changes in subthalamic nucleus activity are not associated with alterations in firing rate or pattern in layer $\mathrm{V}$ neurons of the anterior cingulate cortex in anesthetized rats. Eur J Neurosci 26:1925-1939. CrossRef Medline

Parr-Brownlie LC, Poloskey SL, Bergstrom DA, Walters JR (2009) Parafascicular thalamic nucleus activity in a rat model of Parkinson's disease. Exp Neurol 217:269-281. CrossRef Medline

Pasquereau B, Turner RS (2011) Primary motor cortex of the parkinsonian monkey: differential effects on the spontaneous activity of pyramidal tract-type neurons. Cereb Cortex 21:1362-1378. CrossRef Medline

Paxinos G, Watson C (2007) The rat brain in stereotaxic coordinates, Ed 6. London: Elsevier.

Perret E (1968) Simple motor performance of patients with Parkinson's disease before and after a surgical lesion in the thalamus. J Neurol Neurosurg Psychiatry 31:284-290. CrossRef Medline

Perret E, Eggenberger E, Siegfried J (1970) Simple and complex finger movement performance of patients with Parkinsonism before and after a unilateral stereotaxic thalamotomy. J Neurol Neurosurg Psychiatry 33: 16-21. CrossRef Medline

Person AL, Perkel DJ (2005) Unitary IPSPs drive precise thalamic spiking in a circuit required for learning. Neuron 46:129-140. CrossRef Medline

Person AL, Perkel DJ (2007) Pallidal neuron activity increases during sensory relay through thalamus in a songbird circuit essential for learning. J Neurosci 27:8687-8698. CrossRef Medline

Pessiglione M, Guehl D, Rolland AS, François C, Hirsch EC, Féger J, Tremblay L (2005) Thalamic neuronal activity in dopamine-depleted primates: evidence for a loss of functional segregation within basal ganglia circuits. J Neurosci 25:1523-1531. CrossRef Medline

Ramcharan EJ, Gnadt JW, Sherman SM (2005) Higher-order thalamic relays burst more than first-order relays. Proc Natl Acad Sci U S A 102: 12236-12241. CrossRef Medline

Sacrey LA, Alaverdashvili M, Whishaw IQ (2009) Similar hand shaping in reaching-for-food (skilled reaching) in rats and humans provides evi- dence of homology in release, collection, and manipulation movements. Behav Brain Res 204:153-161. CrossRef Medline

Sakai ST, Inase M, Tanji J (1996) Comparison of cerebellothalamic and pallidothalamic projections in the monkey (Macaca fuscata): a double anterograde labeling study. J Comp Neurol 368:215-228. CrossRef Medline

Schlag-Rey M, Schlag J (1984) Visuomotor functions of central thalamus in monkey. I. Unit activity related to spontaneous eye movements. J Neurophysiol 51:1149-1174. Medline

Schmied A, Bénita M, Conde H, Dormont JF (1979) Activity of ventrolateral thalamic neurons in relation to a simple reaction time task in the cat. Exp Brain Res 36:285-300. Medline

Schneider JS, Rothblat DS (1996) Alterations in intralaminar and motor thalamic physiology following nigrostriatal dopamine depletion. Brain Res 742:25-33. CrossRef Medline

Steriade M, Dossi RC, Nuñez A (1991) Network modulation of a slow intrinsic oscillation of cat thalamocortical neurons implicated in sleep delta waves: cortically induced synchronization and brainstem cholinergic suppression. J Neurosci 11:3200-3217. Medline

Strick PL (1976) Activity of ventrolateral thalamic neurons during arm movement. J Neurophysiol 39:1032-1044. Medline

Tai CH, Yang YC, Pan MK, Huang CS, Kuo CC (2010) Modulation of subthalamic T-type $\mathrm{Ca} 2+$ channels remedies locomotr deficits in a rat model of Parkinson disease. J Clin Invest 121:3289-3305. CrossRef Medline

Tanji J, Okano K, Sato KC (1988) Neuronal activity in cortical motor areas related to ipsilateral, contralateral, and bilateral digit movements of the monkey. J Neurophysiol 60:325-343. Medline

Vitek JL, Ashe J, DeLong MR, Alexander GE (1994) Physiologic properties and somatotopic organization of the primate motor thalamus. J Neurophysiol 71:1498-1513. Medline

Voloshin MYu, Lukhanina EP, Kolomietz BP, Prokopenko VF, Rodionov VA (1994) Electrophysiological investigation of thalamic neuronal mechanisms of motor disorders in parkinsonism: an influence of D2ergic transmission blockade on excitation and inhibition of relay neurons in motor thalamic nuclei of cat. Neuroscience 62:771-781. CrossRef Medline

Walters JR, Hu D, Itoga CA, Parr-Brownlie LC, Bergstrom DA (2007) Phase relationships support a role for coordinated activity in the indirect pathway in organizing slow oscillations in basal ganglia output after loss of dopamine. Neuroscience 144:762-776. CrossRef Medline

Weinrich M, Wise SP (1982) The premotor cortex of the monkey. J Neurosci 2:1329-1345. Medline

Weinrich M, Wise SP, Mauritz KH (1984) A neurophysiological study of the premotor cortex in the rhesus monkey. Brain 107:385-414. CrossRef Medline

Whishaw IQ, Pellis SM (1990) The structure of skilled forelimb reaching in the rat: a proximally driven movement with a single distal rotatory component. Behav Brain Res 41:49-59. CrossRef Medline

Whishaw IQ, Pellis SM, Gorny BP (1992) Skilled reaching in rats and humans: evidence for parallel development or homology. Behav Brain Res 47:59-70. CrossRef Medline

Whishaw IQ, Gorny B, Tran-Nguyen LT, Castañeda E, Miklyaeva EI, Pellis SM (1994) Making two movements at once: impairments of movement, posture, and their integration underlie the adult skilled reaching deficit of neonatally dopamine-depleted rats. Behav Brain Res 61:65-77. CrossRef Medline

Whishaw IQ, Suchowersky O, Davis L, Sarna J, Metz GA, Pellis SM (2002) Impairment of pronation, supination, and body co-ordination in reachto-grasp tasks in human Parkinson's disease (PD) reveals homology to deficits in animal models. Behav Brain Res 133:165-176. CrossRef Medline

Wichmann T, DeLong MR (1996) Functional and pathophysiological models of the basal ganglia. Curr Opin Neurobiol 6:751-758. CrossRef Medline

Wilson SAK (1925) Disorders of motility and of muscle tone. Lancet 206:110, 53-62. CrossRef

Zirh TA, Lenz FA, Reich SG, Dougherty PM (1998) Patterns of bursting occurring in thalamic cells during parkinsonian tremor. Neuroscience 83:107-121. CrossRef Medline 